\title{
Computation of vortex sheet roll-up in the Trefftz plane
}

\author{
By ROBERT KRASNY $\dagger$ \\ Courant Institute of Mathematical Sciences, New York University, \\ 251 Mercer St., New York, NY 10012, USA
}

(Received 28 April 1986 and in revised form 20 March 1987)

\begin{abstract}
Two vortex-sheet evolution problems arising in aerodynamics are studied numerically. The approach is based on desingularizing the Cauchy principal value integral which defines the sheet's velocity. Numerical evidence is presented which indicates that the approach converges with respect to refinement in the mesh-size and the smoothing parameter. For elliptic loading, the computed roll-up is in good agreement with Kaden's asymptotic spiral at early times. Some aspects of the solution's instability to short-wavelength perturbations, for a small value of the smoothing parameter, are inferred by comparing calculations performed with different levels of computer round-off error. The tip vortices' deformation, due to their mutual interaction, is shown in a long-time calculation. Computations for a simulated fuselage-flap configuration show a complicated process of roll-up, deformation and interaction involving the tip vortex and the inboard neighbouring vortices.
\end{abstract}

\section{Introduction}

An important problem in aerodynamics is to determine and control the structure of an aircraft's wake. Not only is the wake important because of the induced drag on the generating aircraft, but also because it can pose a hazard for a following aircraft. The physical phenomena involved are complicated and simplifying assumptions must be made in order to achieve progress. The present work studies a commonly used model in which the shed vorticity in the wake is replaced by a vortex sheet, i.e. a surface across which the tangential velocity component is discontinuous. A further simplification is to replace the spatial coordinate in the aircraft's line of flight by a time coordinate. This changes the steady three-space-dimensional free-boundary-value problem into an initial value problem for the vortex sheet's evolution as a curve in two space dimensions (the Trefftz plane). The physical process by which vorticity enters the wake at the wing's trailing edge is accounted for by specifying an initial spanwise circulation distribution along the vortex sheet, corresponding to a particular loading on the wing. Two problems will be considered here : elliptic loading and the loading for a simulated fuselage-flap configuration. For background information on the vortex sheet/Trefftz plane model of an aircraft wake see Batchelor $(1967, \S 7.8)$. Some possibilities and limitations of the model are reviewed by Smith (1986).

The aim of this work is to present a computational method for studying the vortex sheet's evolution. Even though the model under consideration is conceptually simple, the computational difficulties encountered are quite challenging. A numerical method

$\dagger$ Present address: Mathematics Department, The University of Michigan, Ann Arbor, MI 48109, USA. 
must be capable of accurately approximating the roll-up phenomenon, i.e. the formation in the vortex sheet of single- and double-branched spirals having an infinite number of turns. Another computational difficulty is that spurious perturbations introduced by computer round-off error can be amplified in regions where the vortex sheet is unstable. It is hoped that gaining a deeper understanding of these issues in the present context will lead to progress with more realistic wake models.

Kaden (1931) derived an asymptotic expression for the rolling-up spiral that forms at the tip of an elliptically loaded wing (see also Moore 1975; Guiraud \& Zeytounian 1977; and Saffman \& Baker 1979). Pullin (1978) and Pullin \& Phillips (1981) have studied similarity solutions for this tip vortex using numerical methods, obtaining good agreement with Kaden's solution. These studies of asymptotic and self-similar solutions deal with a semi-infinite vortex sheet, in order to focus attention upon the basic roll-up process. Since the interaction between the two tip vortices is neglected, these results are valid for the finite-span evolution problem during a short time interval, before the two tip spirals grow significantly in size. There have also been many previous attempts to compute the time evolution of the finite-span vortex sheet. We shall briefly review some of the work on the elliptically loaded wing problem (see Saffman \& Baker 1979; Hoeijmakers 1983, 1986; and Smith 1986 for further references).

The earliest numerical study was by Westwater (1935), using the point-vortex approximation of Rosenhead (1931). The vortex sheet was approximated by a finite number of point vortices and the ordinary differential equations for the point vortices' motions were integrated numerically in time. Later investigators (Takami 1964 ; Moore 1971) found that using larger numbers of point vortices and more accurate time integration methods did not lead to convergent results for the elliptically loaded wing problem. Instead, the point vortices moved in an irregular manner with the consequence that an interpolating curve drawn through them became tangled and developed self-intersections.

Various approaches have since been investigated in order to obtain more reliable vortex-sheet results. One approach has been to replace the inner turns of the rolling-up spiral by an isolated point vortex. Any other point vortex that moves to within a specified distance of this isolated point vortex is removed from the calculation and its strength is added to that of the isolated point vortex. Moore (1974) showed that such a tip amalgamation procedure removed the small-scale irregular motion of the point vortices. Besides using an isolated point vortex to represent the inner spiral turns, Fink \& Soh (1978) reset the other point vortices' positions at every time-step so that they were equally spaced in chord length, and Hoeijmakers \& Vaatstra (1983) used a second-order panel method to compute the velocity induced by the continuous part of the curve. These studies succeeded in obtaining computed curves that had up to four smooth outer turns of the tip spiral.

Another approach was taken by Chorin \& Bernard (1973) who replaced the point vortex' singular velocity field with a bounded velocity field in a small neighbourhood of the singularity. Kuwahara \& Takami (1973) smoothed the point-vortex velocity field by letting it evolve in time according to the linear diffusion equation. Computations showed that these devices stabilize the calculation although neither work carefully studied the computational accuracy obtained.

The numerical methods mentioned thus far have in common that the computed velocity is obtained by discretizing an integral over the vortex sheet. Another approach has been to use the vortex-in-cell method (Baker 1979; Murman \& Stremel 1982) in which a point vortex' velocity is computed approximately by solving a 
Poisson equation on an underlying regular grid. Interpolation formulas transfer information between the grid and the point vortices. The cost per computational element for this method is significantly less than for the previously mentioned quadrature-based methods and consequently, the vortex sheet may be represented by a larger number of points. However, both studies concluded that while the vortex sheet's large-scale properties could be correctly computed by this method, the fine structure was computed inaccurately.

The present computational work seeks to study vortex-sheet evolution with greater accuracy and for longer times than has been achieved in the previous investigations. The approach taken is a refinement of the ideas expressed by Chorin \& Bernard (1973). The key element in our computational approach is to desingularize the Cauchy principal value integral which defines the velocity of a Lagrangian point on the vortex sheet. The desingularized evolution equation is characterized by a smoothing parameter and the exact vortex-sheet evolution equation is recovered when that parameter is set to zero. DiPerna \& Majda $(1987 a, b)$ have recently begun a rigorous mathematical study of such limit processes. Numerical evidence will be presented here indicating that solutions of the desingularized equation converge to the vortex sheet as the smoothing parameter converges to zero. Outside a neighbourhood of the vortex sheet's spiral centre, the convergence is uniform and the error behaves like an asymptotic series in powers of the smoothing parameter.

The purpose of introducing the smoothing parameter is to make the problem computationally tractable. One effect of this desingularization is that the infinite spiral vortex sheet is approximated by a continuous curve having only a finite number of turns. As opposed to the exact solution, such an approximating curve can be well resolved using a sufficiently large, but finite, number of computational elements. By reducing the smoothing parameter, more turns are represented and the rolling-up spiral can be more closely approximated, within the limits imposed by the available computing resources. This desirable situation contrasts with what occurs when using the straightforward point-vortex approximation for the present problem (Takami 1964; Moore 1971). In that case, refining the computational mesh does not lead to a better approximation of the vortex sheet. The desingularization used here also effectively diminishes the vortex sheet's short-wavelength instability. An important practical consequence is that the computational inaccuracy arising from the growth of short-wavelength perturbations introduced spuriously by computer round-off error can be controlled. The harmful effect that round-off error can have upon the computational accuracy will be demonstrated.

Previous numerical studies have not carefully examined the issue of computational accuracy. The present work will numerically demonstrate that, at a fixed moderate time, the desingularization approach converges with respect to refinement in the mesh and smoothing parameters. Several calculations, using relatively large values of the smoothing parameter, will also be presented which follow the evolution to times at which, due to computational expense, the numerical convergence has not been extensively documented. In these cases, we shall strive to make the effect of the mesh size negligibly small. These results therefore depend upon the specific value of the smoothing parameter used in the calculation. It is fair to question the extent to which such long-time results describe the vortex sheet's actual evolution. The long-time results should therefore be viewed cautiously. It is believed that they correctly reflect large-scale properties of the vortex sheet such as overall spiral shapes, sizes and relative positions. However it is not known to what extent they accurately describe the small-scale structure. The long-time results are presented with the hope of 

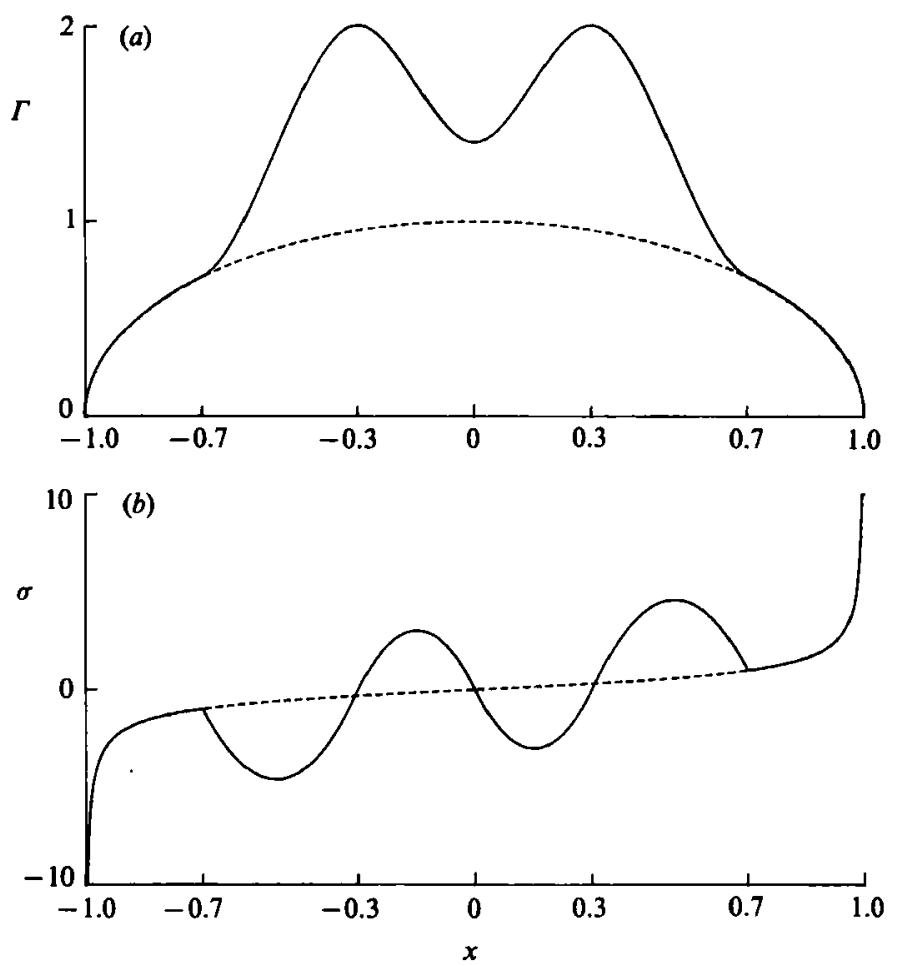

Figure 1. (a) Circulation $\Gamma$, (b) initial vortex-sheet strength $\sigma$ (plotted as functions of the spanwise coordinate $x$ ). Dashed line: elliptically loaded wing; solid line: simulated fuselage-flap configuration.

stimulating thought and providing possible clues about the true nature of vortex-sheet evolution.

The paper is organized as follows. The governing equations and numerical method are presented in $§ 2$. Computations for the elliptically loaded wing and the simulated fuselage-flap configuration are given in $\$ 3$ and $\S 4$. The results are discussed and conclusions are given in $\$ 5$.

\section{Problem description and numerical method}

\subsection{Governing equations}

The vortex sheet is defined by a curve $z(\Gamma, t)=x(\Gamma, t)+\mathrm{i} y(\Gamma, t)$ in the complex plane ( $x$ is the spanwise coordinate). The variable $\Gamma$ is a Lagrangian parameter along the curve which measures the circulation contained between a base point and any other point on the sheet. The variable $t$ is time. The vortex-sheet strength $\sigma=-\mathrm{d} \Gamma / \mathrm{d} s$, where $s$ is the arclength, is the jump in the tangential component of velocity across the sheet. For the problems considered here, it is convenient to make a change of variable $\Gamma=\Gamma(\alpha)$, where $0 \leqslant \alpha \leqslant \pi$. The conditions $\Gamma(0)=\Gamma(\pi)=0$ will be imposed so that the values $\alpha=0, \pi$ correspond to the wing tips. The evolution equation for the vortex sheet is

$$
\frac{\partial \bar{z}}{\partial t}=\int_{0}^{\pi} K(z-\tilde{z}) \Gamma^{\prime}(\tilde{\alpha}) \mathrm{d} \tilde{\alpha}
$$

(Birkhoff 1962; Rott 1956). In this equation $z=z(\Gamma(\alpha), t), \tilde{z}=z(\Gamma(\tilde{\alpha}), t)$ and $K(z)=-1 / 2 \pi \mathrm{i} z$ is the Cauchy kernel (Stein \& Weiss 1971). The slash on the integral 
sign indicates that the Cauchy principal value of the integral is taken. The bar over the time derivative on the left denotes the complex conjugate. The vortex sheet's initial condition is $z(\Gamma(\alpha), 0)=-\cos \alpha$ so that it coincides with the straight line segment $-1 \leqslant x \leqslant 1$. The problem's definition is completed by specifying the circulation distribution $\Gamma(\alpha)$.

Figure 1 shows the circulation $\Gamma$ and the corresponding initial vortex-sheet strength $\sigma$ plotted as functions of the spanwise coordinate $x$ for the two problems studied in this paper. The first problem is for elliptic loading,

$$
\Gamma(\alpha)=\sin \alpha .
$$

In this case, the initial velocity $\partial z / \partial t$ of a point on the vortex sheet is $-\frac{1}{2} \mathrm{i}$ if $|x|<1$. One might conclude from this that for $t>0$, the vortex sheet translates steadily downward without changing shape from its initial flat state. Note however that the initial vortex sheet strength $\sigma=x\left(1-x^{2}\right)^{-\frac{1}{2}}$ has algebraic singularities at $x= \pm 1$. On the horizontal axis $y=0$, the sheet's velocity becomes infinite as $x \rightarrow 1$ from above and as $x \rightarrow-1$ from below. Also, it is commonly accepted that for $t>0$ the two ends of the vortex sheet roll-up into counter-rotating spirals. This suggests the possibility of non-uniqueness of solutions to this initial-value problem. Presumably the rollingup vortex sheet is the physically relevant solution although the existence of such a solution has not yet been rigorously proven.

The second problem to be studied here uses elliptic loading for $0.7 \leqslant|x| \leqslant 1$ but has a circulation distribution away from the wing tips which simulates the presence of a fuselage and deployed flaps. In each interval $0 \leqslant|x| \leqslant 0.3$ and $0.3 \leqslant|x| \leqslant 0.7$, $\Gamma$ is defined to be a cubic polynomial in $|x|$. The polynomials' coefficients are chosen so that $\Gamma$ and $\sigma$ are continuous at the points $x=0, \pm 0.3, \pm 0.7$. Finally, there is a maximum value $\Gamma=2$ at $|x|=0.3$ and a local minimum value $\Gamma=1.4$ at $x=0$. Donaldson, Snedeker \& Sullivan (1974) asserted that for each local maximum that is present in the absolute value $|\sigma|$ of the initial vortex-sheet strength, a spiral forms during the sheet's evolution. Besides the single-branched spiral at each tip, doublebranched spirals form inboard, taking their sense of rotation from the sign of the initial vortex-sheet strength at the local maximum of $|\sigma|$. This problem was studied numerically by Baker (1979), Murman \& Stremel (1982) and Hoeijmakers \& Vaatstra (1983).

\subsection{Desingularization}

In preparation for desingularizing the vortex-sheet equation, we consider a more familiar singular integral operator, the Hilbert transform $H f(x)$ of a function $f(x)$,

$$
H f(x)=\lim _{\varepsilon \rightarrow 0} \int_{|x \rightarrow \varepsilon| \geqslant \varepsilon} Q_{0}(x-s) f(s) \mathrm{d} s=\lim _{y \rightarrow 0} \int Q_{y}(x-s) f(s) \mathrm{d} s .
$$

The kernel $Q_{y}(x)=x / \pi\left(x^{2}+y^{2}\right)$ is called the conjugate Poisson kernel. The first limit in (3) is the definition of the integral's Cauchy principal value. The second limit expresses $H f(x)$ as the boundary value of a function which is harmonic in the upper half-plane $\{(x, y): y>0\}$. Both expressions are useful in studying the Hilbert transform's properties. The point in mentioning this is that the second expression provides heuristic motivation for considering a desingularized approximation to the vortex-sheet evolution equation (1).

Let $\delta>0$ be an artificial smoothing parameter and consider the ' $\delta$-equation',

$$
\frac{\partial \bar{z}}{\partial t}=\int_{0}^{\pi} K_{\delta}(z-\tilde{z}) \Gamma^{\prime}(\tilde{\alpha}) \mathrm{d} \tilde{\alpha}
$$


where $K_{\delta}(z)=K(z)\left(|z|^{2} /|z|^{2}+\delta^{2}\right)$. Since $K_{\delta}(0)=0$ if $\delta>0$, the integrand vanishes when $\tilde{\alpha}=\alpha$ and the integral is proper. For a given value of $\delta>0$ the solution of the $\delta$-equation (4) is a curve which approximates the vortex sheet. As $\delta \rightarrow 0$ the desingularized kernels $K_{\delta}(z)$ converge to the Cauchy kernel $K(z)$ for $z \neq 0$. It may therefore be expected that the solution of the $\delta$-equation converges to the solution of the vortex-sheet equation (1). Numerical evidence supporting this hypothesis will be presented below. This issue has also recently been studied for a periodic vortex sheet (Krasny 1986 $b$ ) and for an interface separating fluids of slightly different densities (Anderson 1985).

\subsection{Discretization}

Straightforward discretization techniques will be applied to solve the $\delta$-equation numerically. Let $z_{j}(t)=x_{j}(t)+\mathrm{i} y_{j}(t)$ be an approximation to the vortex sheet's exact position $z\left(\Gamma\left(\alpha_{j}\right), t\right)$ at equidistant parameter values $\alpha_{j}=\pi(j-1) / 2 N, j=1, \ldots, 2 N+1$. The integral in (4) is approximated by the trapezoidal rule, yielding a system of ordinary differential equations for the points' motion,

$$
\frac{\mathrm{d} \overline{z_{j}}}{\mathrm{~d} t}=\sum_{k=1}^{2 N+1} K_{\delta}\left(z_{j}-z_{k}\right) w_{k} .
$$

The quadrature weights are defined by $w_{k}=\Gamma^{\prime}\left(\alpha_{k}\right) \pi / 2 N, k=1, \ldots, 2 N+1\left(w_{1}\right.$ and $w_{2 N+1}$ have the appropriate extra $\frac{1}{2}$ factor). The initial point positions are given by $z_{j}(0)=-\cos \alpha_{j}$. If $\delta$ is set to zero and the $k=j$ term is omitted from the sum, the system (5) describes the evolution of a set of point vortices. For $\delta>0$ the basic computational element is called a 'vortex blob' since it amounts to making a smooth approximation of a point vortex. Our computations used the fourth-order RungeKutta method with a fixed time-step $\Delta t$ to integrate the real and imaginary parts of the system (5).

The computations took advantage of several symmetries in order to reduce the computational expense. The problems studied are symmetric about $\alpha=\frac{1}{2} \pi$, allowing a reduction by $\frac{1}{2}$ in the number of computational elements. A further factor of $\frac{1}{2}$ was gained by using the fact that $K_{\delta}\left(z_{j}-z_{k}\right)=-K_{\delta}\left(z_{k}-z_{j}\right)$. The computations were performed on VAX 8600 and CRAY computers. Vectorizing the code for the CRAY was straightforward.

As occurs for point vortices, the vortex-blob equations (5) form a Hamiltonian system with the Hamiltonian function given by

$$
H=\sum_{k>j} w_{j} w_{k} \ln \left(\left|z_{j}-z_{k}\right|^{2}+\delta^{2}\right)^{\frac{1}{2}}
$$

In the computations, the invariance of $H$ was used to check the time integration's accuracy. Another invariant of the point-vortex equations is

$$
X=\frac{\sum_{j=1}^{N+1} x_{j} \omega_{j}}{\sum_{j=1}^{N+1} \omega_{j}}
$$

the lateral position of the centre of circulation on each side of the symmetry plane $x=0$. This quantity is also invariant when $\delta>0$ and in our calculations $X$ was conserved to more significant digits than was $H$. It should be noted that both $H$ and $X$ as defined above are discretizations of analogous conserved integrals of the continuous $\delta$-equation (4) for $\delta \geqslant 0$. 
We emphasize that the calculations necessarily use a finite set of different values for $N, \Delta t$ and $\delta$. Since we are interested in the vortex sheet we shall draw conclusions, based on this collection of numerical experiments, about the limiting behaviour when $N \rightarrow \infty, \Delta t \rightarrow 0$ and $\delta \rightarrow 0$. Some of these conclusions will be supported by extensive numerical documentation of the limiting behaviour. Other conclusions about the vortex sheet, based upon a small number of long-time calculations with a relatively large value of the smoothing parameter, are more speculative since they have not been thoroughly validated, owing to the restrictions imposed by computing resources. However, care was taken to ensure that the discretization error in these long-time calculations is negligible. This narrows the uncertainty to the only remaining approximation, namely the effect of using a non-zero value for the smoothing parameter $\delta$.

The strategy adopted is to fix a value of $\delta$ and then to choose the mesh parameters $\left(N^{-1}\right.$ and $\Delta t$ ) small enough so as to obtain an accurate solution of the continuous $\delta$-equation (4) over the desired time interval. Previous investigators (Moore 1971; Takami 1964) found that point-vortex calculations for the elliptic loading problem do not converge as the mesh parameters are refined. By contrast, it will be demonstrated that for fixed $\delta>0$, numerical solutions of the vortex-blob equations (5) do converge under mesh refinement to a smoothly rolling-up curve. Furthermore, the solutions obtained for different values of $\delta$ converge to a limit curve as $\delta \rightarrow 0$. This limit curve can then be interpreted as a weak solution of the original vortex-sheet evolution equation (1).

\section{The elliptically loaded wing}

\subsection{Early stage of the roll-up}

Figure 2 shows the computed curve's evolution over the time interval $0 \leqslant t \leqslant 4$ for the elliptically loaded wing problem. The value $\delta=0.05$ was used for the smoothing parameter, with the values $N=200$ and $\Delta t=0.01$ for the mesh parameters. It will be demonstrated shortly that this numerical solution of the $\delta$-equation (4) has converged to within plotting accuracy with respect to refinement in the mesh parameters $N$ and $\Delta t$. This computation was performed in single-precision arithmetic and took less than half an hour to run on a VAX 8600 computer. The vortex-blob positions are plotted on the left side of figure 2 and a trigonometric interpolating polynomial in the variable $\alpha$ is plotted on the right side. The vortex-blob positions $z_{j}$ approximate the vortex sheet at the equally spaced parameter values $\alpha_{j}$ and the coefficients of the interpolating polynomial are simply the discrete Fourier cosine coefficients of the $z_{j}$.

The curve in figure 2 rolls-up smoothly, forming two counter-rotating spirals each with 13 complete turns at time $t=4$. Plotting the interpolating curve helps to bring out details of the computed solution which may not be apparent from the vortex-blob positions alone. Each tip vortex travels upward for a short time, until the far-field effect of the other tip vortex becomes strong. Thereafter, the entire spanwise structure moves downward, propagating roughly like a vortex dipole. As time progresses, there is also a slow drift inward of each rolling-up core, toward the symmetry line $x=0$. Both the horizontal and vertical dimensions of the curve increase with time, as does the curvature at the midpoint $x=0$. 


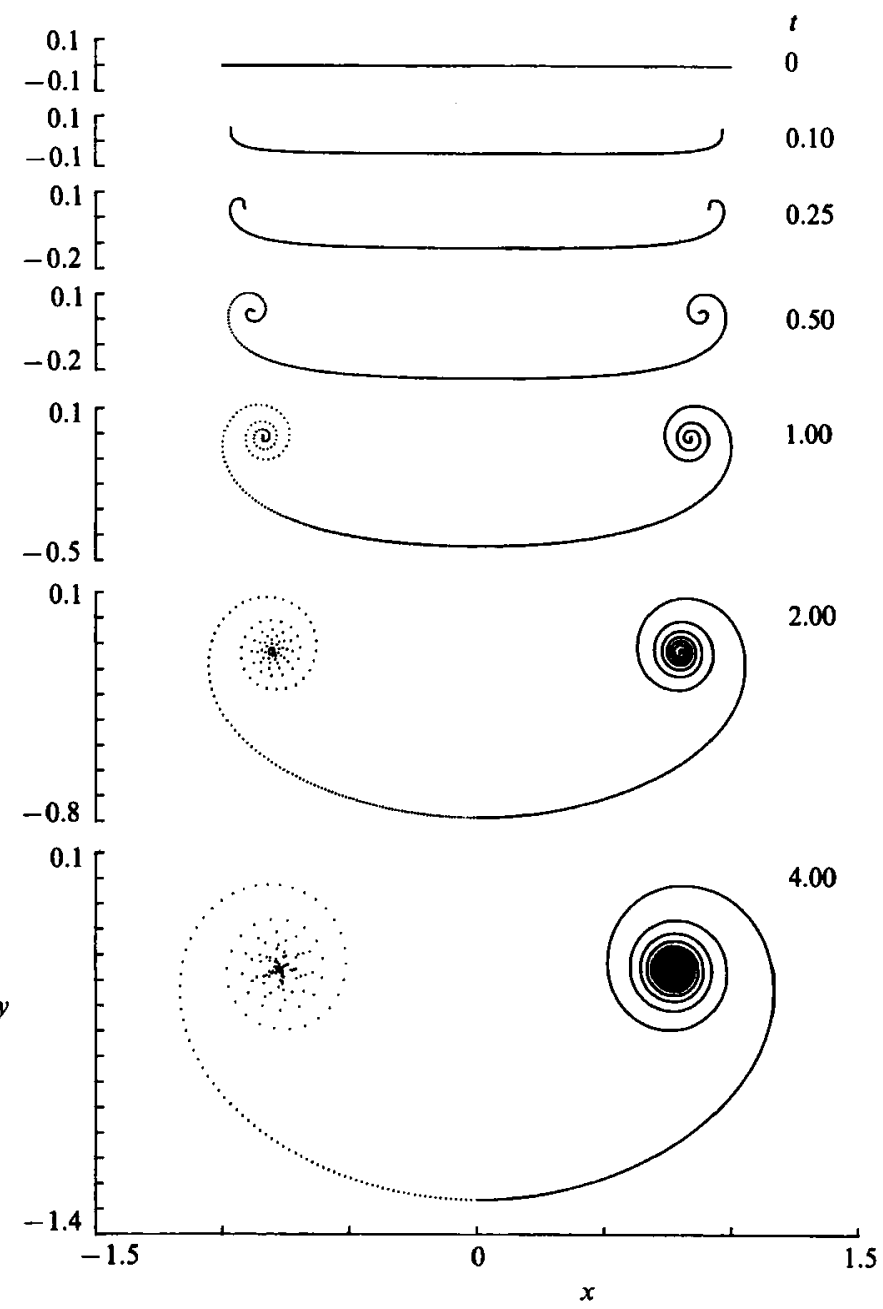

Figure 2. Early stage of the roll-up $(0 \leqslant t \leqslant 4)$. Time sequence of the solution computed using $\delta=0.05, N=200$ and $\Delta t=0.01$. The vortex-blob positions are plotted on the left and an interpolating curve is plotted on the right.

\subsection{Convergence with respect to mesh refinement}

Figure 3 shows a closeup of the interpolating curve in the tip region at time $t=4$ for computations using values of $N$ equal to 50,100 and 200. The time-step for these calculations $(\Delta t=0.01$ as in figure 2$)$ was sufficiently small that the timediscretization error in the plotted curves is negligible. It is clear that the solution converges as the value of $N$ increases, with the curve's outer turns converging fastest. Each frame in figure 3 has a small plus sign $(+)$, a circle $(O)$ and a box $(\square)$ drawn at the computed curve positions corresponding to the three parameter values $\alpha=\frac{3}{4} \pi, \frac{13}{16} \pi$ and $\frac{7}{8} \pi$. More detailed information about the convergence at these representative values of $\alpha$ is given in figure 4 which plots the computed $x$-coordinate as a function of $N^{-1}$ for several values of $N$ in the range $40 \leqslant N \leqslant 800$. The convergence is non-monotonic as $N$ increases. The computed $x$-coordinates have already converged to within three significant digits when $N=200$. 


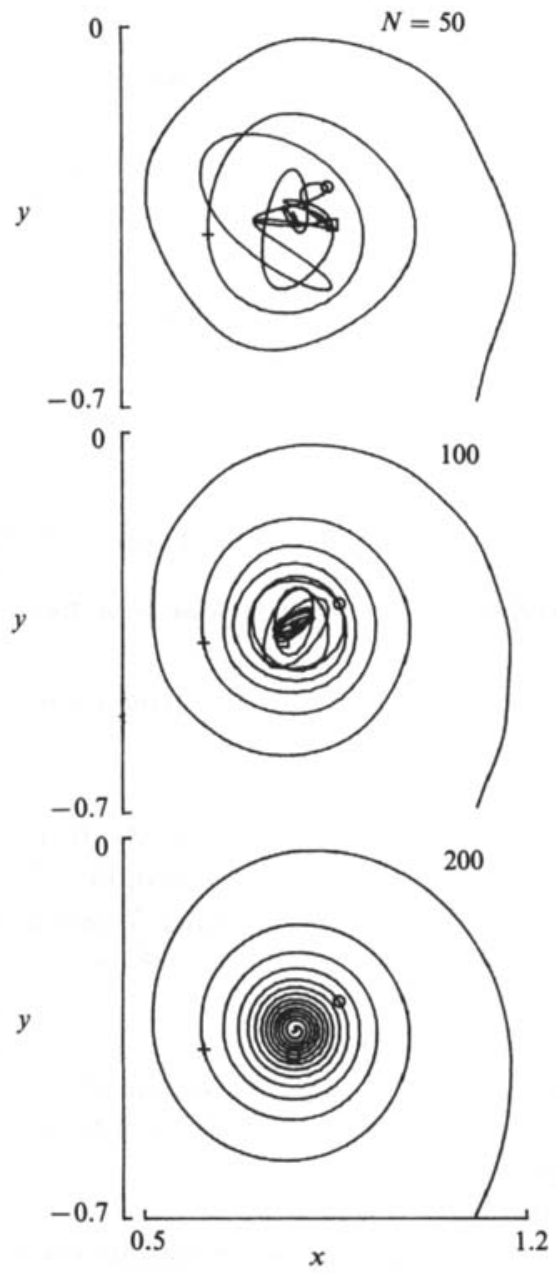

Figure 3. Convergence as $N \rightarrow \infty$. Closeup view of the tip region at $t=4$. The interpolating curve is plotted for computations using $N=50,100$ and 200 . The values $\delta=0.05$ and $\Delta t=0.01$ were used, as in figure $2 .+, \alpha=\frac{3}{4} \pi ; 0, \frac{18}{16} \pi ; \square, \frac{7}{8} \pi$.

The effect of changing the time-step is shown in figure 5 which plots the interpolating curves at $t=4$ that were computed using $\Delta t=0.16,0.08$ and 0.04 . A smooth curve appears as the time-step is reduced, with the outer turns converging fastest. The value $N=200$ was used in each case so that, as already noted, the $\alpha$-discretization error in the plotted curves is negligible. The Hamiltonian values $H_{\Delta t},(6)$, that were computed using several choices of $\Delta t$ are plotted in figure $6(a)$ over the time interval $0 \leqslant t \leqslant 4$. For large values of $\Delta t$, the computed Hamiltonian decays significantly in time. However, with $\Delta t=0.01$ (as in figure 2), $H_{\Delta t}$ is conserved to machine precision. The increase in the computed values of $H_{0.16}$ for $t>0.5$ is not significant; figure $6(b)$ shows that for $\Delta t<0.16$, the computed values $H_{\Delta t}$ at $t=4$ converge like $(\Delta t)^{4}$, the asymptotic order of accuracy for the Runge-Kutta method.

Figures 3-6 establish the numerical method's ability to accurately solve the $\delta$-equation (4) for a fixed value of $\delta>0$. Maintaining a specified level of accuracy for longer times or with a smaller value of $\delta$ is more expensive, requiring larger $N$ and smaller $\Delta t$. Care was taken to ensure that the results presented in the remainder 


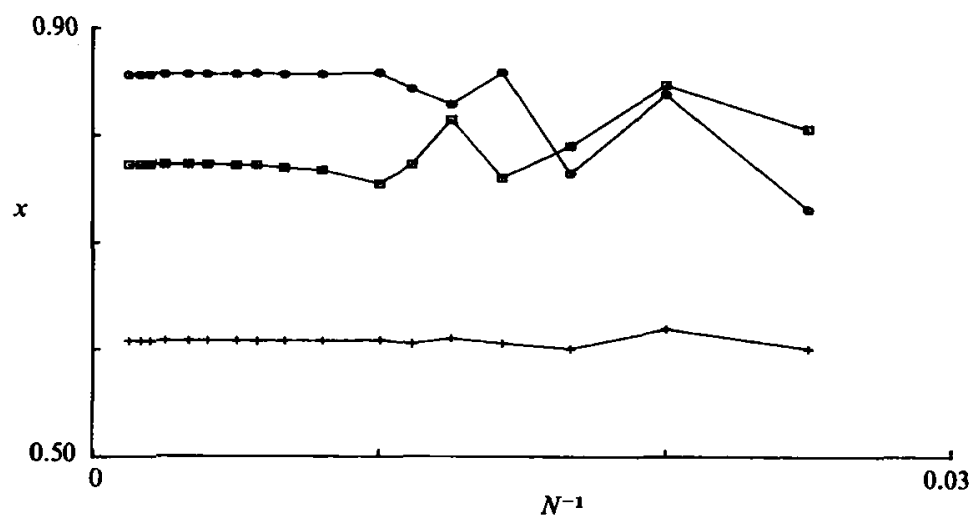

Fiaure 4. The $x$-coordinate of the computed curve at $t=4$ corresponding to the three parameter values $\alpha=\frac{3}{4} \pi(+), \alpha=\frac{13}{16} \pi(O)$ and $\alpha=\frac{7}{8} \pi(\square)$ plotted as a function of $N^{-1}$ in the range $40 \leqslant N \leqslant 800$. The values $\delta=0.05$ and $\Delta t=0.01$ were used, as in figure 2 .

of this paper have negligible discretization error in time $t$ and in the curve parameter $\alpha$.

\subsection{Convergence with respect to the smoothing parameter}

In order to document the method's convergence as $\delta \rightarrow 0$, results were obtained at $t=4$ over the range of smoothing parameter values $0.03 \leqslant \delta \leqslant 0.4$. Views of the tip region for $\delta=0.2,0.1$ and 0.03 are plotted in figure 7 (see also $\delta=0.05$, plotted in figure 3 ). The solution is most sensitive to $\delta$ in a neighbourhood of the tip where there is a small 'hook', similar to that seen in the work of Murman \& Stremel (1982). The number of complete turns in the computed curve increases as $\delta$ becomes smaller. Figure 8 shows a closeup view of the $\delta=0.03$ case in which 30 complete turns are present. This calculation used the values $N=1200$ and $\Delta t=0.01$, and took 17 hours of c.p.u. time to run in single-precision arithmetic on a VAX 8600 computer.

Data was collected for values of $\delta$ in the interval $0.05 \leqslant \delta \leqslant 0.4$. Owing to the high computational expense of resolving the solution for small values of $\delta$, only two smaller values, $\delta=0.04$ and $\delta=0.03$, were also computed. Using this set of computed curves, detailed information about the nature of convergence as $\delta \rightarrow 0$, at the fixed time $t=4$, is given in figures $\mathbf{9 - 1 1}$.

Figure 9 contains information about how the overall size of the computed curve depends on $\delta$. Figure $9(a)$ plots the curves' maximum $x$-coordinate $\left(x_{\max }\right)$ and the maximum and minimum $y$-coordinates $\left(y_{\max }, y_{\min }\right)$, as functions of $\delta$. Figure $9(b)$ plots the $\alpha$-values $\left(\alpha_{x_{\max }}, \alpha_{y_{\max }}\right)$ at which the maximum $x$-and $y$-coordinates are attained (the $\alpha$-value corresponding to $y_{\min }$ is not plotted since, by symmetry, it always occurs at $\alpha=\frac{1}{2} \pi$ ). Nothing is plotted for $y_{\max }$ and $\alpha_{y_{\max }}$ in the interval $0.35 \leqslant \delta \leqslant 0.4$ because, with such large values of the smoothing parameter, the computed curve did not contain a complete turn at $t=4$. The small circles in figure 9 are the data values for $0.03 \leqslant \delta \leqslant 0.4$, in intervals of $\Delta \delta=0.01$. The solid lines in figure 9 are quadratic polynomials in $\delta$ whose coefficients were determined by least-squares fitting to the data values. The horizontal and vertical dimensions of the computed solution increase monotonically as $\delta$ becomes smaller. It may be inferred from the good fit obtained that limiting values of these various quantities exist as $\delta \rightarrow 0$ and that the convergence is like an asymptotic power series in $\delta$, e.g.

$$
x_{\max }(\delta)=x_{\max }(0)+c_{1} \delta+c_{2} \delta^{2}+\ldots
$$




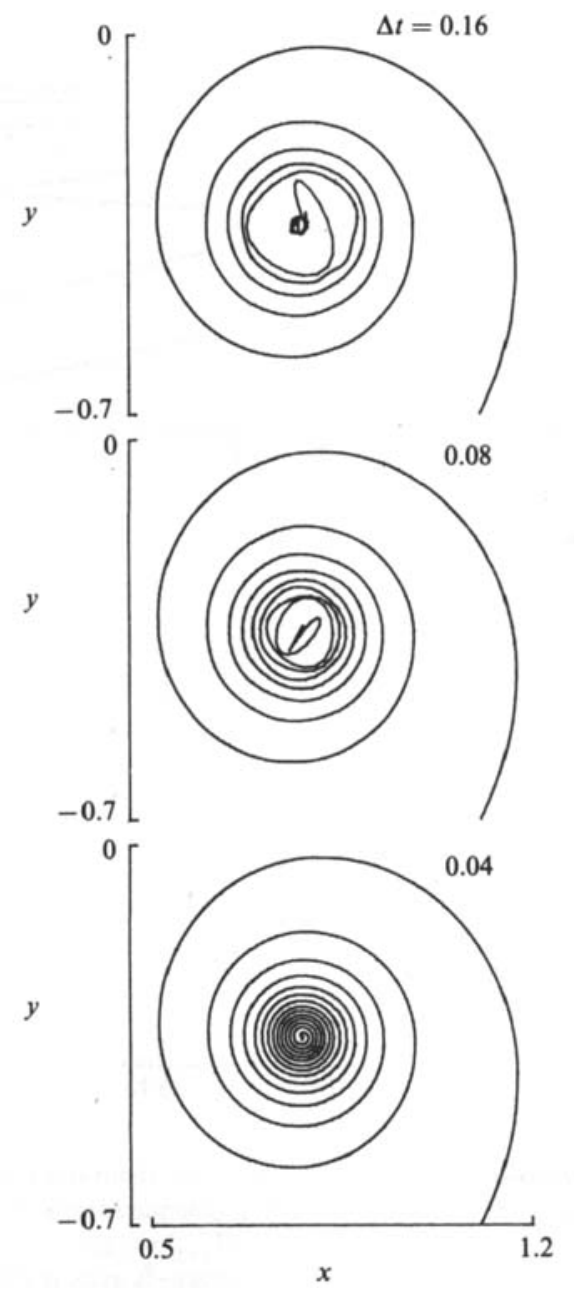

Figure 5. Convergence as $\Delta t \rightarrow 0$. Closeup view of tip region at $t=4$. The interpolating curve is plotted for computations using $\Delta t=0.16,0.08$ and 0.04 . The values $\delta=0.05$ and $N=200$ were used, as in figure 2.

In particular, the limit curve's outer dimensions are fairly insensitive to the precise value of $\delta$. Similar convergence was also observed in a study of a periodic vortex sheet (Krasny $1986 b$ ).

Figure 10 shows the variation with $\delta$ of the computed $x$-coordinates for the three curve parameter values $\alpha=\frac{3}{4} \pi(+), \frac{13}{16} \pi(O)$ and $\frac{7}{8} \pi(\square)$. The data values in each frame have been connected by a straight line interpolant for clarity. Some of the corresponding physical points have already been plotted in figure 3 and figure 7 . The oscillations appearing in figure 10 are due to the fact that for fixed $\alpha$, the corresponding physical point travels around the endpoint $\alpha=\pi$ as $\delta$ decreases. For large enough $\delta$, the curve has no turns at all (the right side of the $\delta$-equation (4) vanishes as $\delta$ becomes large). As $\delta$ is reduced, more turns appear in the curve and the physical point corresponding to a fixed value of $\alpha$ appears on successive turns. The extrema of the oscillations in figure 10 occur when the computed curve has a vertical tangent since this is the condition that the physical point's $x$-coordinate 

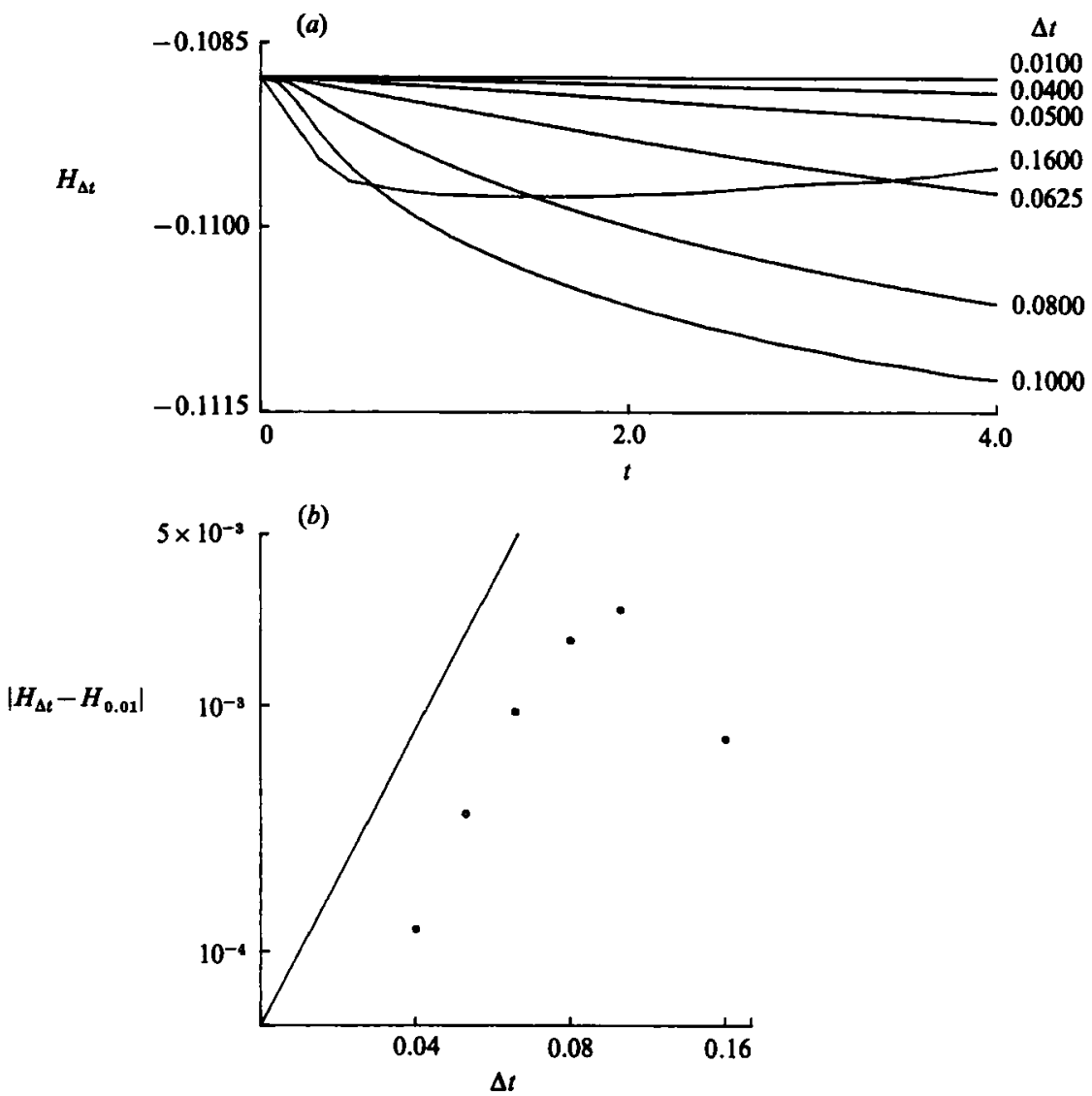

Figure 6. (a) Values of the Hamiltonian $H_{\Delta t},(6)$, plotted vs. time over the interval $0 \leqslant t \leqslant 4$, for computations performed with several values of $\Delta t$. These computations used $\delta=0.05$ and $N=200$. (b) Log-log plots of the computed error at $t=4,\left|H_{\Delta t}-H_{0.01}\right|$ vs. $\Delta t$. The straight line has slope 4, the asymptotic convergence rate for the fourth-order Runge-Kutta method.

changes direction. Thus for each value of $\alpha$ in figure 10, the number of complete cycles in the graph is also the number of the turn, counting from the outermost turn, on which the physical point is located. For example, with $\alpha=\frac{13}{16} \pi$ (figure 10b) there are 5 complete cycles and the physical point, denoted by a circle $(O)$, is located on the fifth turn of the $\delta=0.03$ solution in figure 8. A related feature is that the oscillation amplitude in figure 10 becomes smaller as $\alpha$ increases. This is because larger values of $\alpha$ are closer to $\pi$, the computed curve's endpoint, and hence are located on inner turns of smaller radius. For each value of $\alpha$, the oscillations have a characteristic wavelength $\lambda$ which also depends upon $\delta$. If the wavelength $\lambda$ vanished with the smoothing parameter $\delta$, it would imply that the physical point travels infinitely often around the endpoint. This cannot occur if the physical point ultimately lies on a particular turn of the limit spiral. Consequently, it is proposed that for each curve parameter value $0<\alpha<\pi$, the oscillation wavelength $\lambda$ remains strictly positive as $\delta \rightarrow 0$.

Figure 11 plots, as a function of $\delta,(a)$ the $x$-coordinates (to the right of the endpoint $\alpha=\pi)$ at which the curves have a vertical tangent and $(b)$ the corresponding $\alpha$-values. Only the eight outermost turns are included in this plot since too few data points 


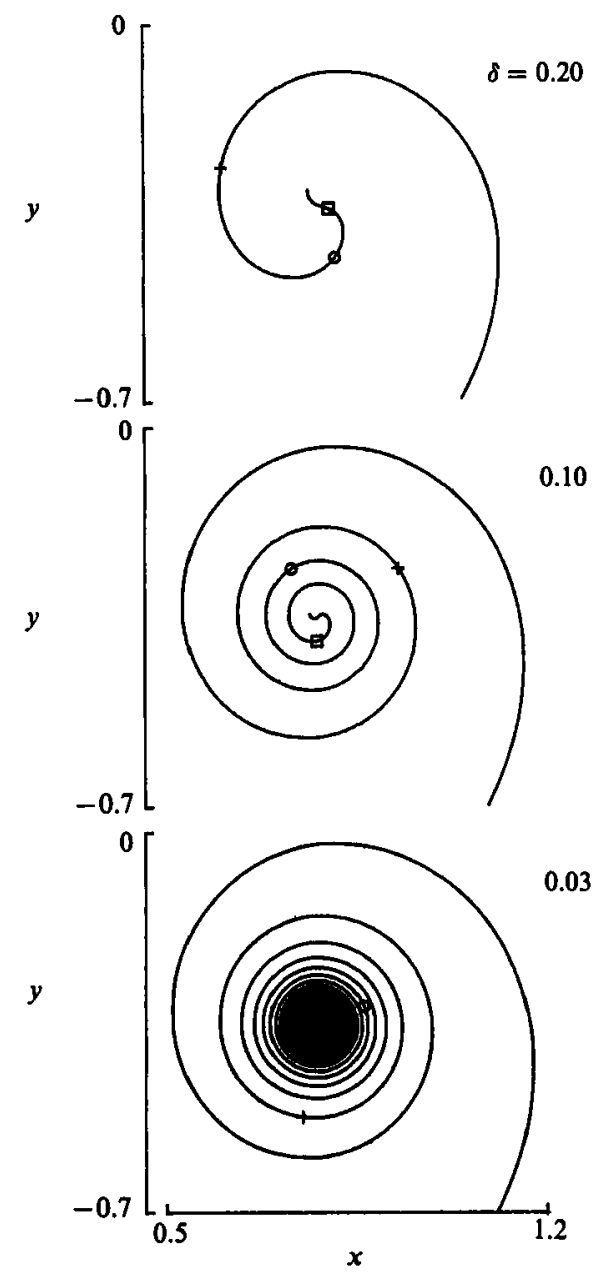

Fraure 7. Views of the tip region at $t=4$ for smoothing parameter values $\delta=0.2, \delta=0.1$ and $\delta=0.03 .+, \alpha=\frac{3}{4} \pi ; O, \frac{18}{18} \pi ; \square, \frac{7}{8} \pi$.

were available for the inner turns. Again, small circles show the data values for $0.03 \leqslant \delta \leqslant 0.4$, in steps of $\Delta \delta=0.01$, and the solid lines are least-squares quadratic polynomials in $\delta$. The good fit obtained indicates that, away from the spiral centre, the error due to smoothing behaves like an asymptotic series in powers of $\delta,(8)$. The convergence of the $x$-coordinates in figure 11 is monotonic and occurs faster away from the limit spiral's centre.

Qualitatively similar behaviour in the limit $\delta \rightarrow 0$ was observed at other times. To illustrate this, closeup views in figure 12 show the tip region at $t=0.1$, over the range $0.003 \leqslant \delta \leqslant 0.015$. The $\delta=0.003$ solution contains 23 turns. Except for the smaller scale, figure 12 resembles the longer time, larger $\delta$ results in figure 7 . In the next subsection we use the $\delta=0.003$ calculation to examine some aspects of the solution's instability to short-wavelength perturbations.

\subsection{Instability for a small value of $\delta$}

The $\delta=0.003$ computation shown in figure 12 at $t=0.1$ used $N=2000$ and $\Delta t=0.0005$. We reiterate that the discretization error in this solution is negligible. 


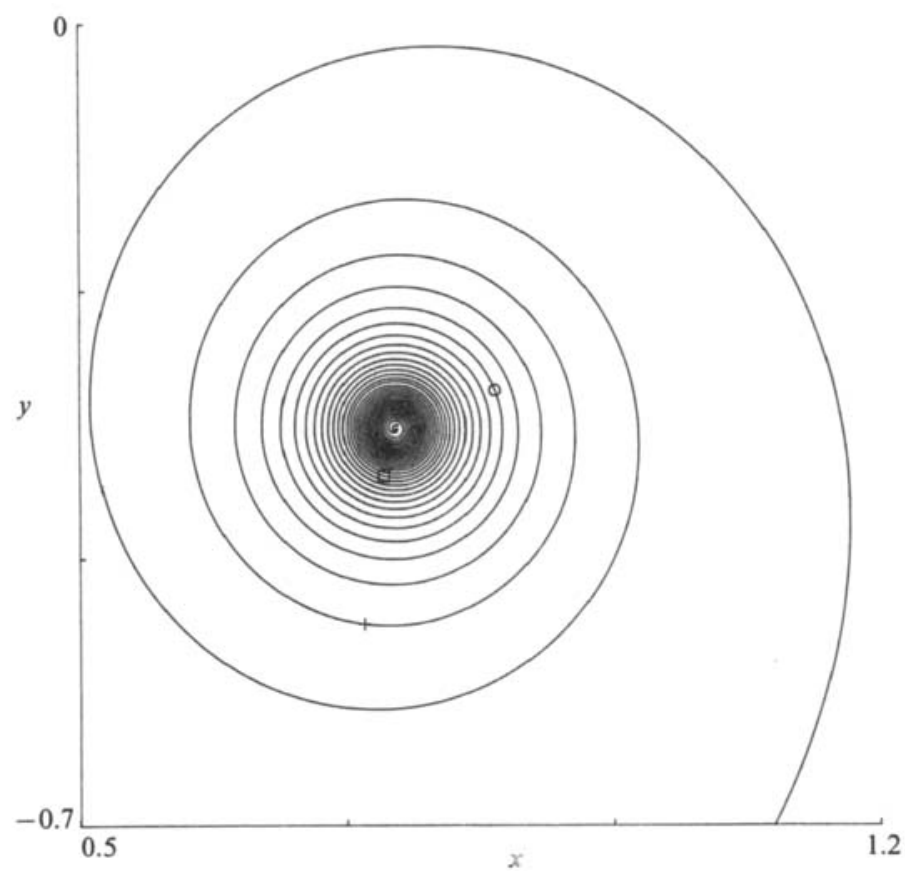

Figure 8. Closeup view of the tip region at $t=4$ for $\delta=0.03$. Thirty complete turns are present.,$+ \alpha=\frac{3}{4} \pi ; O, \frac{18}{16} \pi ; \square, \frac{7}{8} \pi$.

However, as the value of the smoothing parameter is reduced, the solution becomes less stable and as a result, spurious short-wavelength perturbations introduced by computer round-off error can amplify and damage the computational accuracy. Examining these inaccurate computations actually yields useful information about the genuine solution's stability. This is illustrated in figure 13 which compares the results obtained using 7-digit arithmetic (VAX single precision) and 14-digit arithmetic (CRAY single precision). The VAX 8600 calculation took 23 hours of c.p.u. time and the CRAY1 calculation took less than half an hour. The computed curves for $0 \leqslant x \leqslant 0.7$ were quite flat and so only the interval $0.7 \leqslant x \leqslant 1$ is plotted in figure 13. Short-wavelength, small-amplitude waves appear on the curve's outer turn in the 7-digit computation, but they are absent from the 14-digit result. If the single-precision computation in figure $13(a)$ was allowed to proceed, the waves would roll-up into small double-branched spirals and concurrently, these structures would be convected into the core.

A similar phenomenon occurred in computations for a periodically perturbed vortex sheet (Krasny $1986 a, b$ ). The computer's finite-precision arithmetic introduces spurious perturbations into the calculation at the amplitude of the round-off error. These perturbations are amplified in the region where the solution is unstable, leading eventually to the appearance of waves as in figure $13(a)$. In the periodic case, it was shown using linear stability analysis of a flat constant-strength vortex sheet that for each value of $\delta>0$, there is a maximum growth rate $\approx \delta^{-1}$ for a wave of wavelength $\approx \delta$. Depending on the perturbation amplitude (i.e. the round-off unit) and the time interval over which the solution is computed, these waves may destroy the calculation's accuracy. This source of computational error should be distinguished from discretization (or 'truncation') error whose effect was examined in figures 3-6. 

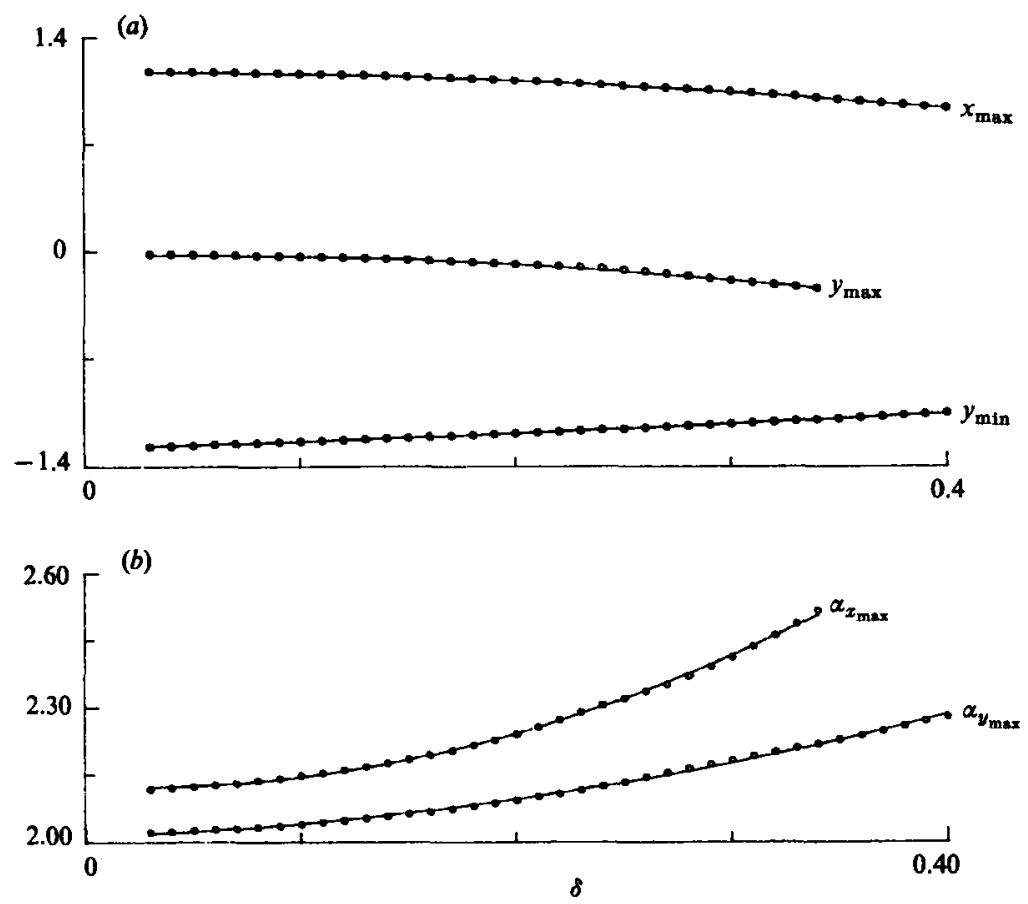

Figure 9. Dependence on the smoothing parameter $\delta$ at $t=4$. (a) Extreme values $x_{\max }, y_{\max }, y_{\min }$. (b) Corresponding $\alpha$-values $\alpha_{x_{\max }}, \alpha_{y_{\max }}$. The $\alpha$-value corresponding to $y_{\min }$ is not drawn since it is always $\alpha=\frac{1}{2} \pi$, owing to symmetry. The small circles are the computed values for $0.03 \leqslant \delta \leqslant 0.4$, in steps of $\Delta \delta=0.01$. The solid lines are quadratic polynomials in $\delta$ whose coefficients were determined by least-squares fitting to the data values.

It is also interesting to examine the solution's 'vorticity' $\omega(z, t)$. If $z$ denotes a point in the complex plane, the expression on the right side of the $\delta$-equation (4) defines the complex-conjugate velocity $(u-\mathrm{i} v)(z, t)$. For $\delta>0$, this velocity field is not a solution of the Euler equations, but it is divergence-free. It is natural to define the approximate vorticity by

$$
\omega(z, t)=\left(\frac{\partial v}{\partial x}-\frac{\partial u}{\partial y}\right)(z, t)=\frac{-\delta^{2}}{\pi} \int_{0}^{\pi}\left(|z-\tilde{z}|^{2}+\delta^{2}\right)^{-2} \Gamma^{\prime}(\tilde{\alpha}) \mathrm{d} \tilde{\alpha} .
$$

The scalar quantity $\omega$ is related to the circulation $\Gamma$ around a closed curve by Green's theorem, $\Gamma=\int u \mathrm{~d} x+v \mathrm{~d} y=\iint \omega \mathrm{d} x \mathrm{~d} y$. Figure $14(a)$ is a contour plot of the computed $\omega$-values for the $\delta=0.003$ solution. A log-linear plot of $\omega$ vs. $x$ on the horizontal line through the core's centre is shown in figure 14(b). The global maximum value $\omega \approx 500$ occurs at the core's centre and there are local peaks in $\omega$ on the curve's outer turns. The magnitude of $\omega$ decreases rapidly away from the core. The smoothness of $\omega$ in figure 14 is due to the integration by which it is defined. Note also from the definition that as $\delta \rightarrow 0, \omega(z, t)$ either becomes infinite or vanishes, depending upon whether or not $z$ lies on the limit curve.

Moore (1974) found that an instability similar to the one in figure $13(a)$ can occur for point-vortex/tip-amalgamation computations. He quoted a suggestion of Professor P. G. Saffman that the instability occurs near a local maximum of the vortex-sheet strength. Our results are consistent with this. In figure $14(a)$ the magnitude of $\omega$ on the curve has a local maximum in the interval $0.75 \leqslant x \leqslant 0.95$, 

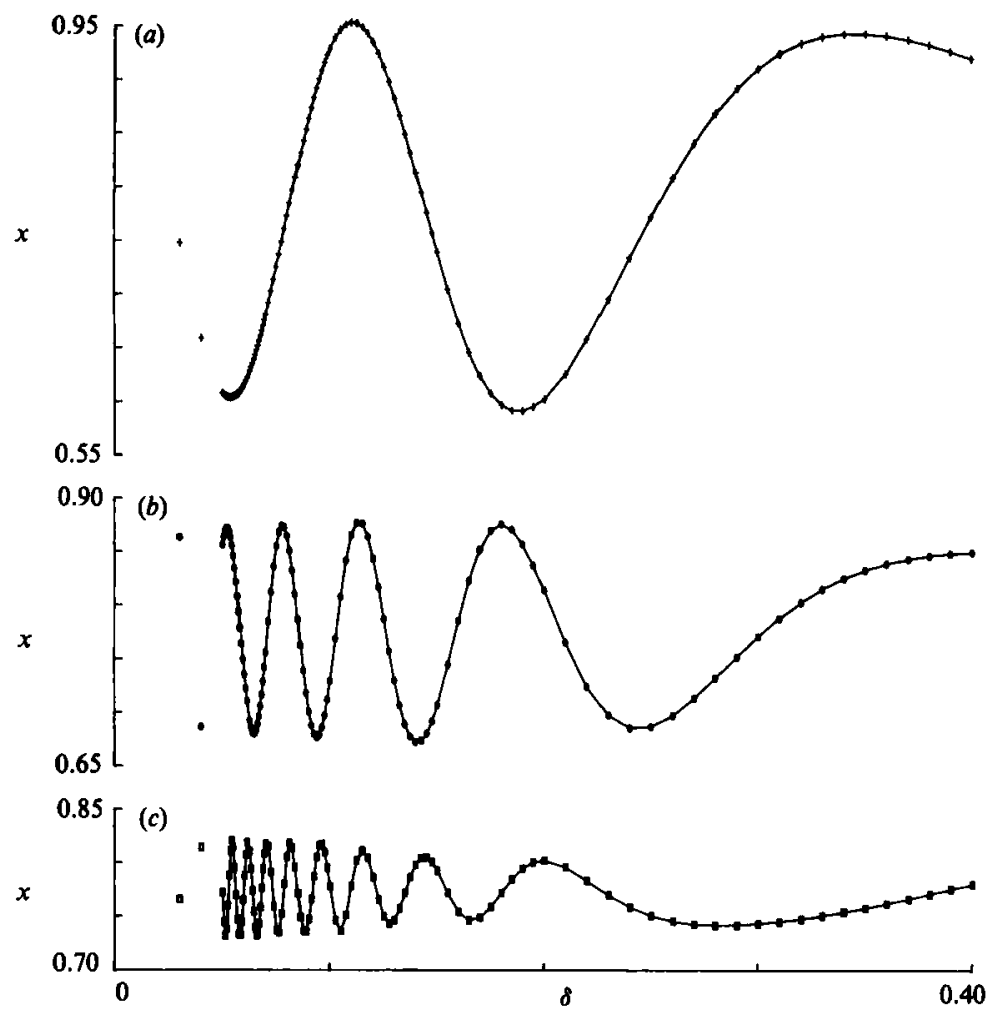

Figure 10. Dependence on the smoothing parameter $\delta$ at $t=4$. The $x$-coordinate of the physical point corresponding to three fixed values of $\alpha$ is plotted. (a),$+ \alpha=\frac{3}{4} \pi ;(b) \bigcirc, \alpha=\frac{18}{16} \pi ;(c) \square, \alpha=\frac{7}{8} \pi$. The small symbols in each frame are the computed values over the interval $0.03 \leqslant \delta \leqslant 0.4$. A straight line interpolant was drawn for clarity.

away from the rolled-up core (note the narrow contour region at level $\omega=25$ in this $x$-interval). An analysis by Moore (1976) indicates that the rolling-up spiral is linearly stable to small perturbations. Close examination of the core in figure 13(a) showed no spurious waves, thus providing support for Moore's conclusion.

\subsection{Comparison with Kaden's solution}

We shall compare the solution computed using $\delta=0.003$ with the following aspects of Kaden's spiral. If $f$ denotes the fraction of circulation contained at time $t$ between the spiral's centre and the outermost point of vertical tangency then $f \approx t^{\frac{1}{3}}$ (see Moore 1974). At any instant of time, Kaden's spiral is described by $\Gamma \approx(2 \lambda r)^{\frac{1}{2}}$ and $r \approx \theta^{-\frac{2}{3}}$, where $r$ is the radial distance from the spiral's centre, $\Gamma$ is the circulation contained between the spiral's centre and the point at radius $r$, and $\theta$ is the polar angle. The constant $\lambda$ cannot be obtained from Kaden's analysis, but Pullin (1978) gave the value $\lambda \approx 2$ based upon numerical solutions for the semi-infinite, self-similar vortex sheet. In deriving Kaden's expressions, the effect of one core on the other is neglected (see Moore \& Saffman 1973 for a simple derivation). Therefore, our comparison is performed for $t \leqslant 0.1$, when the interaction between the two tip vortices can be expected to be small.

The computed circulation fraction $f v s$. time $t$ is plotted on log-log scales in figure $15(a)$. Also plotted is a straight-line segment of slope $\frac{1}{3}$, the exponent from Kaden's expression. Figures $15(b$ and $c)$ pertain to the computed curve at time 

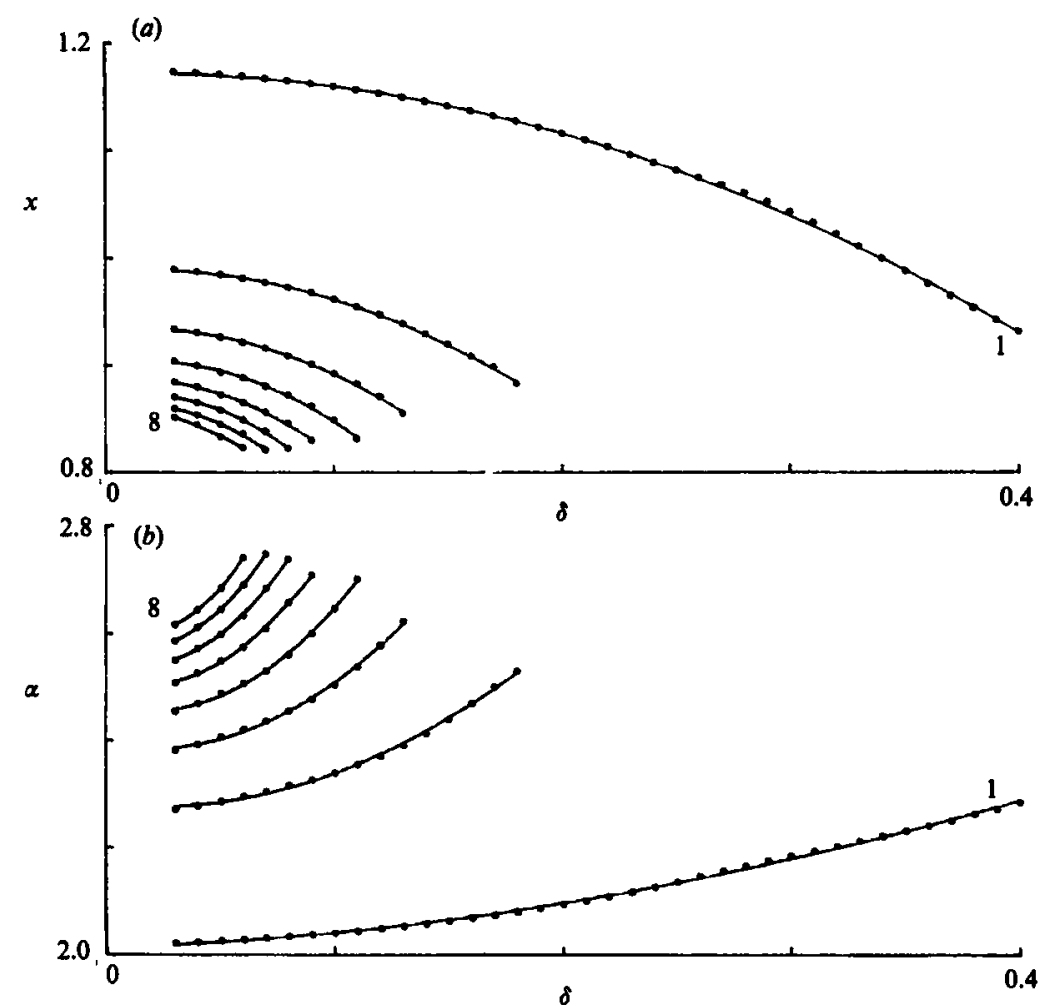

Figure 11. Dependence on the smoothing parameter $\delta$ at $t=4$. (a) $x$-coordinates to the right of the endpoint $\alpha=\pi$ at which the curve has a vertical tangent. (b) Corresponding $\alpha$-values. The small circles are the computed values for $0.03 \leqslant \delta \leqslant 0.4$, in steps of $\Delta \delta=0.01$. The solid lines are least-squares quadratic polynomials in $\delta$. The numerical 1 refers to the outermost turn and the numeral 8 refers to the eighth inner turn.

$t=0.1$ (see figure $12, \delta=0.003$ ). The turns of the computed curve are visually circular and we would like to determine an approximate 'centre' of the rolled-up region in order to measure the curve's radial coordinate. The tip point $(\alpha=\pi)$ was found to be a poor choice for the centre owing to the presence of the small spurious hook. Instead, two line segments normal to the curve were constructed at horizontal and vertical tangency points for a portion of the curve which is less sensitive to the value of $\delta$. The approximate centre $z_{\mathrm{c}}$ was taken as the intersection of the two normal line segments. This enabled us to compute the curve's radial coordinate, $r_{j}=\left|z_{j}-z_{\mathrm{c}}\right|$.

Log-log plots of the computed circulation $\Gamma$ vs. radius $r$, and radius $r v s$. polar angle $\theta$ are shown in figures $15(b$ and $c)$. The figures omit small values of $r_{j}$ where the solution depends most strongly on $\delta$, and also large values of $r_{\boldsymbol{r}}$ where Kaden's solution is not valid. The computed data presented correspond to the 17 outermost turns of the curve. The straight lines in figures $15\left(b\right.$ and $c$ ) have slopes $\frac{1}{2}$ and $-\frac{2}{3}$ respectively, the exponents from Kaden's expressions.

An estimate for the constant $\lambda$ in the relation $\Gamma \approx(2 \lambda r)^{\frac{1}{2}}$ was obtained as follows. The two vortex-blob indices $j_{1}=120$ and $j_{2}=530$ encompass the 17 outermost turns under consideration (counting $j=1$ as the curve's right endpoint and $j=N+1=2001$ as the curve midpoint, at $x=0$ ). The $\log$-log data in figure $15(b)$ have slope $m \approx 0.508$, obtained using the divided difference associated with $j_{1}$ and $j_{2}$. For each vortex blob $z_{j}$ with index $j_{1} \leqslant j \leqslant j_{2}$, the computed values of $r_{j}$ and $\Gamma_{j}$ 


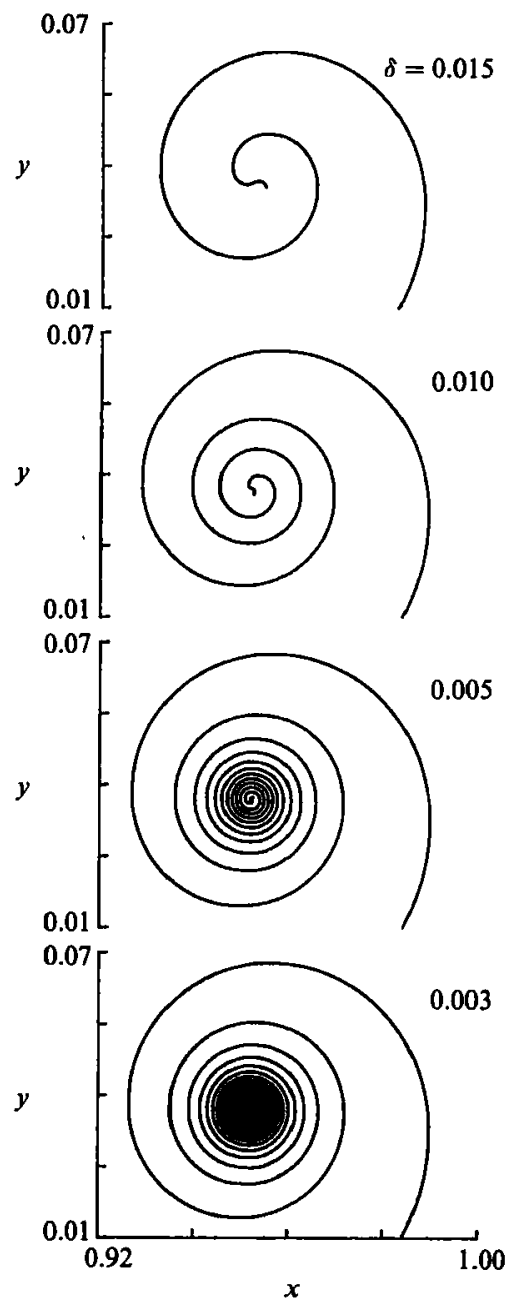

Figure 12. Views of the tip region at $t=0.1$ for several smoothing parameter values in the range $0.003 \leqslant \delta \leqslant 0.015$. There are 23 complete turns present in the $\delta=0.003$ case.

yield an estimate $\lambda_{j}$ via the relation $\Gamma_{j}=\left(2 \lambda_{j} r_{j}\right)^{m}$. The estimates so obtained satisfied $1.89 \leqslant \lambda_{j} \leqslant 2.08$.

The computed results for the finite-span vortex sheet are in good agreement with Kaden's solution for the exponents, and with Pullin's (1978) result for the constant $\lambda$. The computed radial vs. polar-angle dependence (figure $15 c$ ) behaves like $r \approx \theta^{-C}$ over a large interval with a value of $C$ slightly greater than $\frac{2}{3}$. The small discrepancy with Kaden's expressions and Pullin's result may be due to either the non-zero value of $\delta$ being used, or to the imprecision in determining the coordinates of the computed spiral's centre.

\subsection{Late stage of the roll-up}

Figure 16 shows the results of a long-time calculation computed with the value $\delta=0.2$. The reason for using a relatively large value of $\delta$ is practical; it is expensive to accurately compute solutions for small values of $\delta$ over a long-time interval. It was already noted however that the curve's overall shape at $t=4$ depends continuously on $\delta$ (figure 9). The extent to which this remains true at $t=50$ has not been 


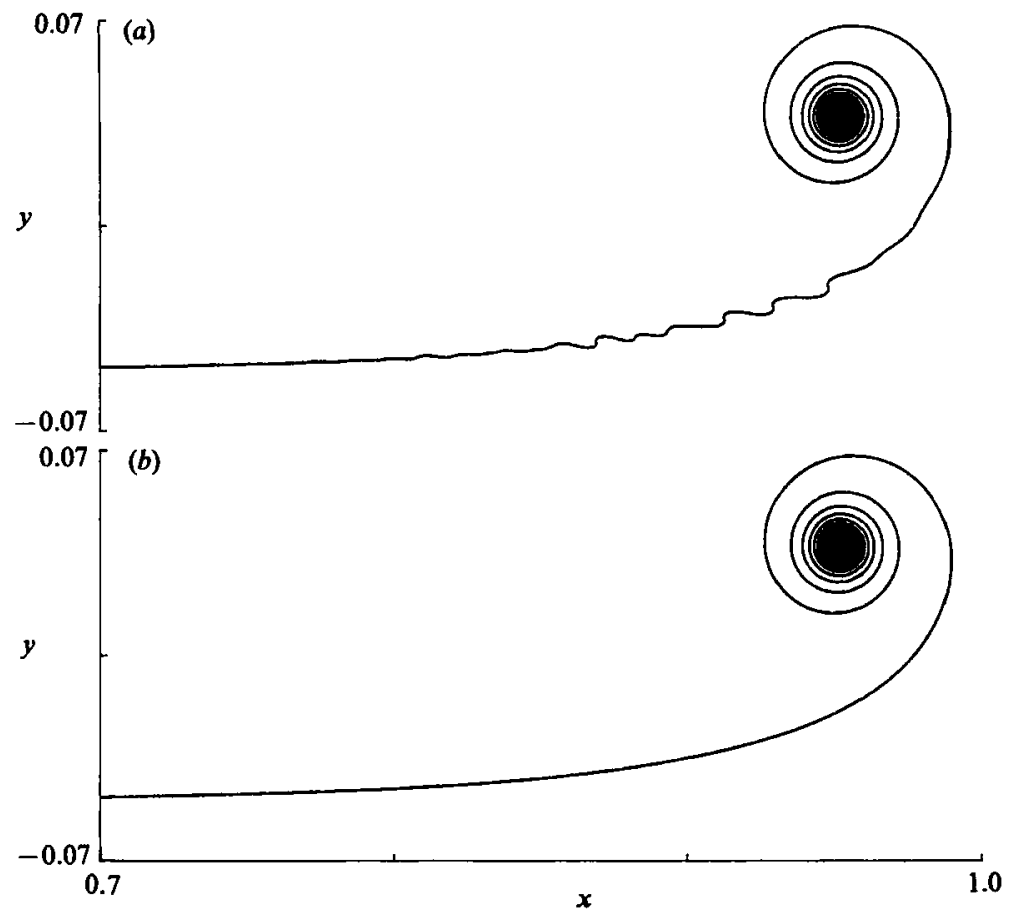

Figure 13. The effect of round-off error. Solution using $\delta=0.003, N=2000$ and $\Delta t=0.0005$, plotted at $t=0.1$. (a) 7-digit arithmetic (VAX single precision). (b) 14-digit arithmetic (CRAY single precision). The interval $0 \leqslant x \leqslant 0.7$ is not plotted since the curves in both calculations were almost flat.

documented. Nonetheless, these results may be useful in giving a hint about the vortex sheet's actual long-time behaviour.

The main features that were observed at early times in figure 2 are continued here in figure 16 - roll-up of the tip regions and propagation downwards of the entire spanwise structure. In figure 16, the cores gradually slow their lateral approach. At $t=50$, the curve's endpoint $\alpha=\pi$ has the $x$-coordinate 0.81 , which is close to the lateral invariant of motion $X \approx 0.79,(7)$. The curve continues to grow in size as it evolves. At late times, the curve portions closest to the line $x=0$ become flattened. The curve's inner turns remain quite circular but the outer turns become non-circular. It is clear from comparing the computational points' separation at consecutive times that the curve's outer turn is highly stretched. At late times, the curve shows some resemblance to the streamline pattern of a steady solution of the two-dimensional Euler equations (Batchelor 1967, \$7.3). Quantifying this observation may be an interesting topic for future study.

\section{The simulated fuselage-flap configuration}

This problem's evolution can be divided, somewhat arbitrarily, into early, middle and late stages, roughly corresponding to increasing degrees of interaction among the vortices in each half-span. The vortices form in the early stage $(0 \leqslant t<1)$, remaining small and well-separated during this time interval. They maintain their individual identity in the middle stage $(1 \leqslant t \leqslant 4)$ but interact more strongly through rotation, propagation and strain-induced elliptical deformation. These interactions continue 


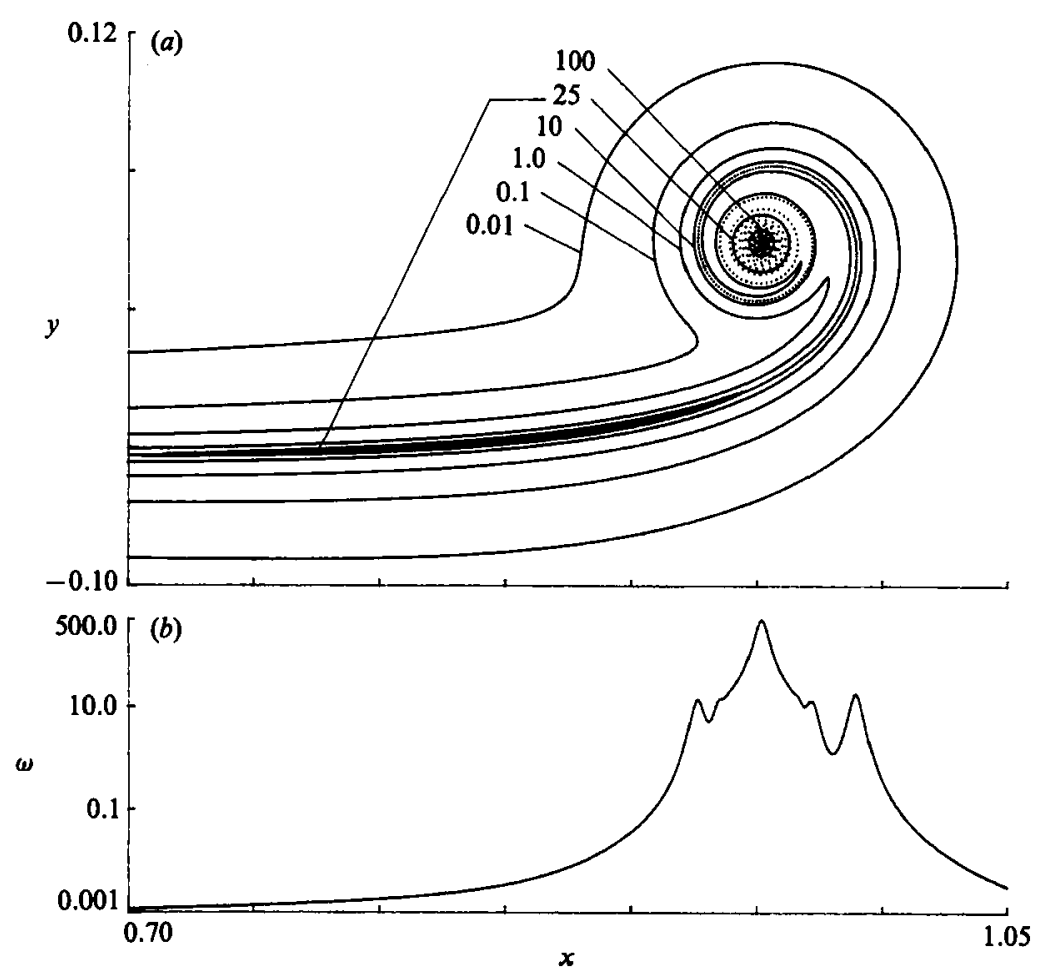

Fraure 14. (a) Contour plot of the computed vorticity $\omega,(9)$, in the tip region of the $\delta=0.003$, $t=0.1$ solution. The vortex-blob positions are also plotted. A thin contour region near the flat part of the curve contains a local maximum at level $\omega \approx 25$. (b) A log-linear plot of $\omega v 8 . x$ on the horizontal line through the core's centre.

into the late stage $(t>4)$ and are then also accompanied by even stronger interactions in which the vortices lose some of their individual identity.

\subsection{Early stage}

Results at $t=0.1,0.3$ and 0.5 for the simulated fuselage-flap configuration are shown in figure 17. A $\delta=0.1$ calculation $(N=200, \Delta t=0.02)$ is shown in figure $17(a)$ and a $\delta=0.02$ calculation $(N=1000, \Delta t=0.002)$ is shown in figure $17(b)$. Three vortices form in each half-span region : a single-branched tip vortex and two double-branched spiral vortices further inboard. Adjacent to the tip vortex, and having the same sense of rotation, is the 'flap vortex'. Further inboard and having the opposite sense of rotation is the 'fuselage vortex'. Such structures were predicted to form by Donaldson et al. (1974). The tip vortex rolls-up first, followed closely in time by the flap vortex, with the fuselage vortex rolling up some time later. At $t=0.5$, the flap vortex is the largest of the three vortices. With $\delta=0.1$, the roll-up proceeds slowly and the fuselage vortex has still not formed at $t=0.5$. By contrast, with $\delta=0.02$ the roll-up is quicker and more of the tightly wound spiral structure is evident. The positions of the vortices are in good qualitative agreement with what has been found by previous numerical investigations (Hoeijmakers \& Vaastra 1983; Murman \& Stremel 1982 ; Baker 1979).

Single- and double-precision computations using the value $\delta=0.005$ are shown in figure 18. The single-precision calculation (figure 18a) shows a clumping of vortex 

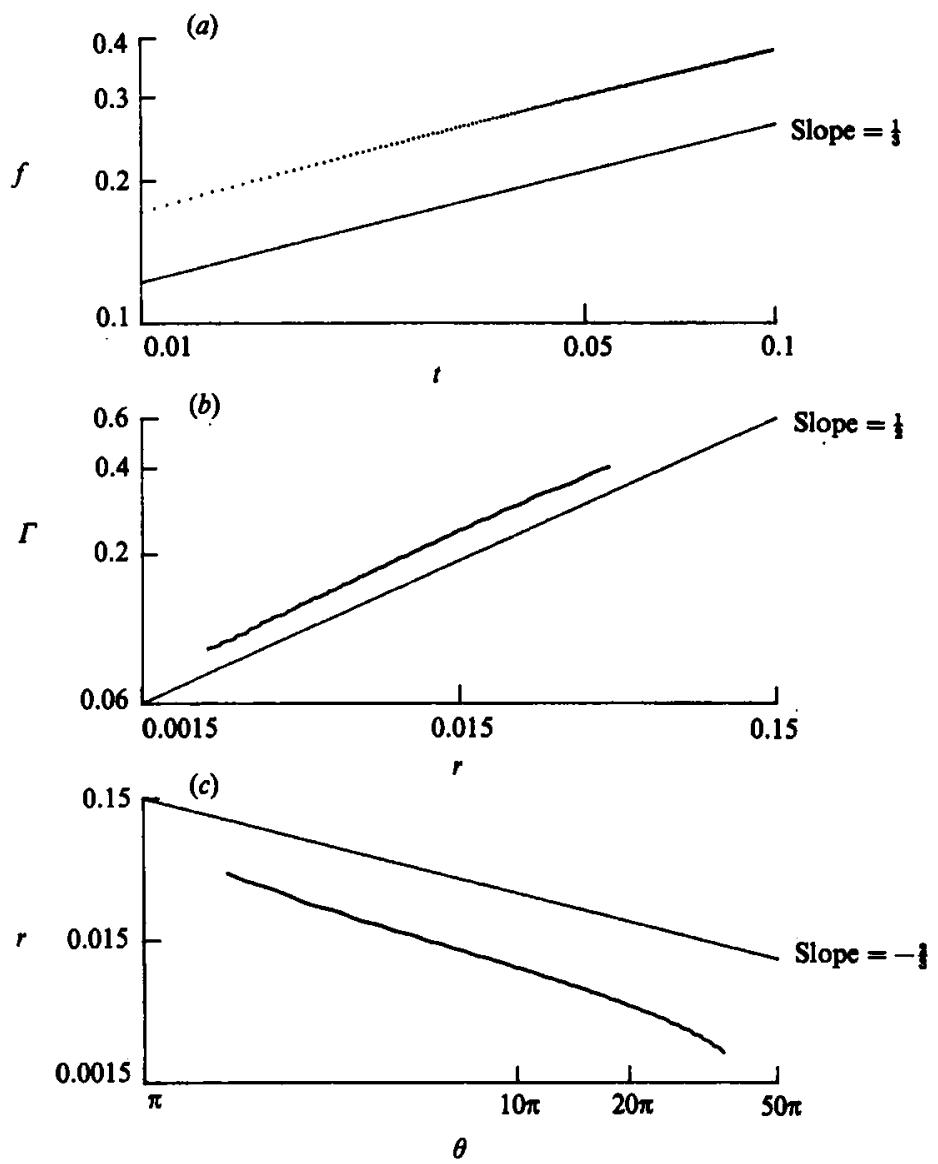

Fiaure 15. Comparison of the $\delta=0.003, t=0.1$ solution with Kaden's asymptotic spiral. Log-log scales are used. (a) The fraction $f$ of rolled-up circulation vs. time for $0.01 \leqslant t \leqslant 0.1$. (b) Circulation $\Gamma v s$. radius $r$ at time $t=0.1$. (c) Radius $r v s$. polar angle $\theta$ at time $t=0.1$. The straight-line segment in each frame has slope given by the exponent in Kaden's expressions. The computed solution in (b) and (c) corresponds to the 17 outermost turns of the curve.

blobs and the formation of spurious waves. Consistent with our previous remarks on instability and round-off error for computations with a small value of $\delta$, the waves are absent from the double-precision calculation (figure 18b). Notice that the flap vortex has begun to roll-up on a small scale at $t=0.1$ unlike in the larger $\delta$ calculations of figure 17. Figure $18(c)$ contains a closeup view of a genuine wave which is believed to roll-up for $t>0.3$ into a double-branched spiral vortex with the same sense of rotation as the tip and flap vortices. The formation of this fourth vortex may be due to the discontinuous slope in the initial vortex-sheet strength at $x= \pm 0.7$ (see figure 1). A fourth vortex was not seen in the previous numerical investigations and it is not predicted by the criterion for vortex formation given by Donaldson $e t$ al. (1974). Since its growth would be inhibited by the stretching which occurs between the tip and flap vortices, the fourth vortex may not significantly affect the dynamics of the solution. Further study of this new phenomenon, though desirable, would strain our present computational resources. 

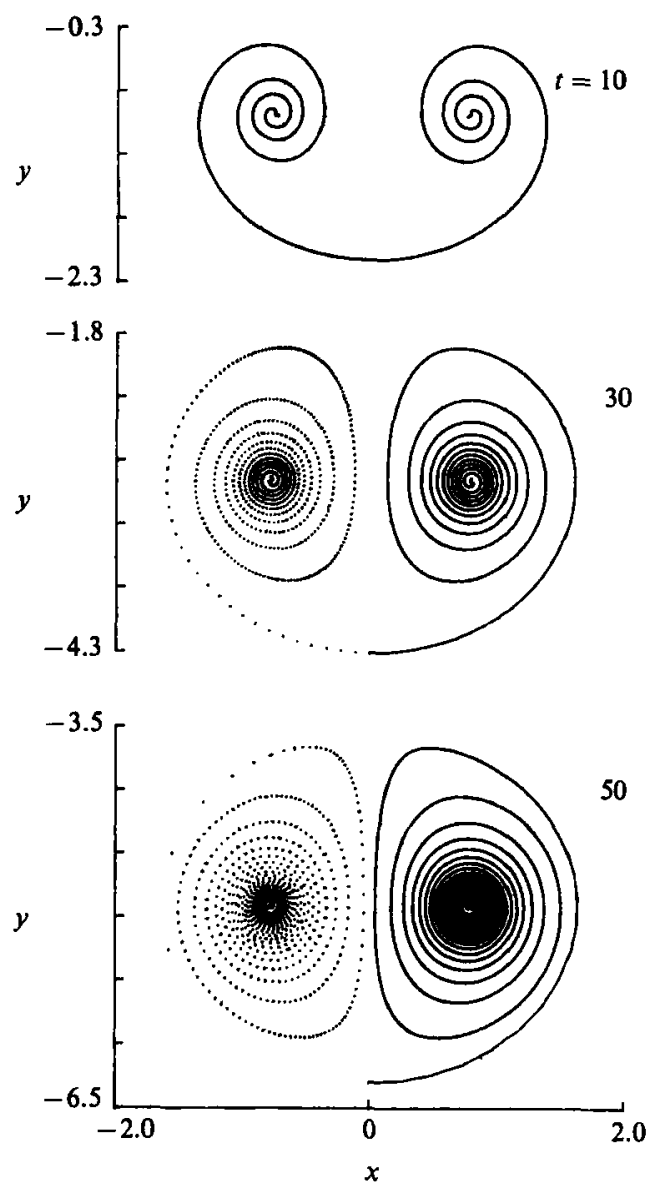

Froure 16. Solution over the time interval $10 \leqslant t \leqslant 50$ using $\delta=0.2$. The vortex-blob positions are plotted on the left and an interpolating curve is plotted on the right.

\subsection{Middle stage}

The values $\delta=0.1$ and $\delta=0.05$ will be used to examine the long-time dynamics. Figure 19 shows the evolution over the time interval $1 \leqslant t \leqslant 4$ with the value $\delta=0.1$. In the closeup views of figure 20 , the portion of the curve which had negative (positive) initial vortex-sheet strength is plotted with a dashed (solid) line. Each closeup view in figure 20 is a square with side 1.5 units, shifted so that the vortices are centred in the plot. This calculation used an adaptive point insertion technique that will be described below. The number of vortex blobs was initially $N=200$ and it had reached $N=971$ at $t=4$.

The dynamics in this stage may be heuristically explained by analogy with simpler vortex structures. The tip and flap vortices rotate around each other as expected for like-signed vortices. There is a tendency for the structure in each half-span to propagate as a vortex dipole due to the presence of oppositely rotating vortices. The fuselage vortex, even though it has the opposite sense of rotation, also tends to rotate around the stronger flap vortex. The combination of rotation and propagation causes the structures to spread laterally more than occurred for the elliptically loaded wing. As time progresses, each vortex undergoes elliptical deformation due to the strain field induced by its neighbours. 
(a)
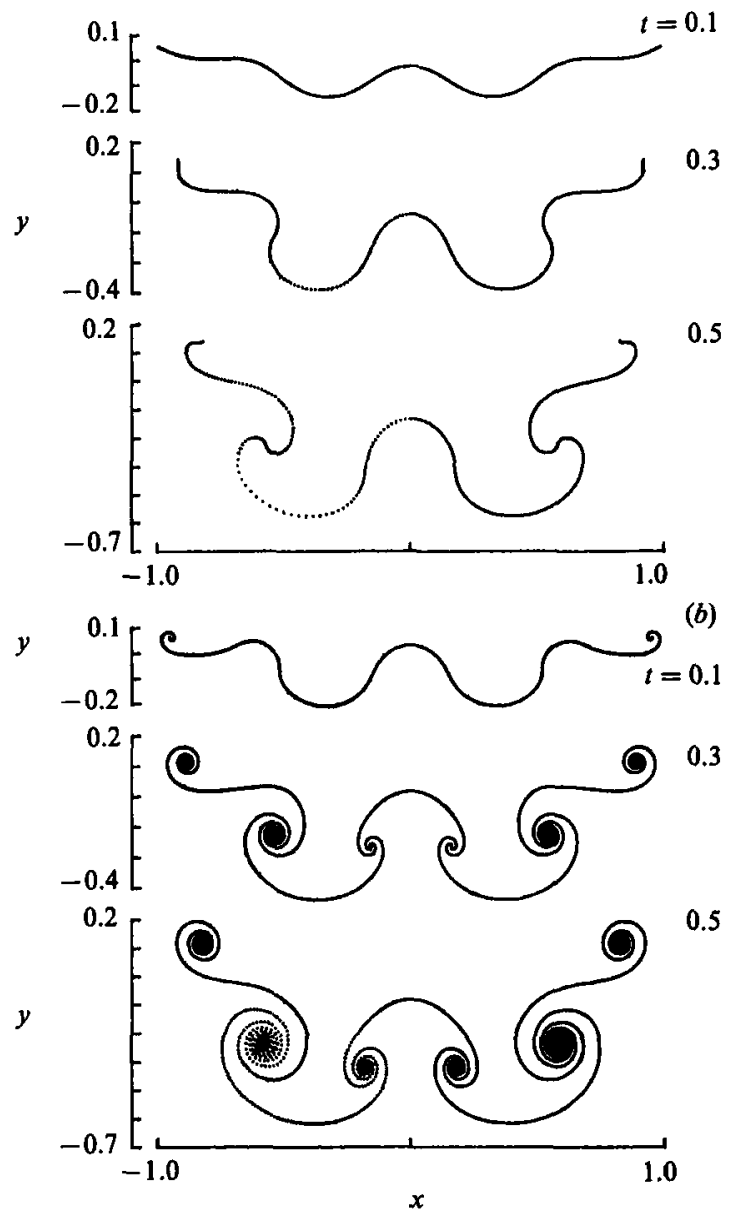

Fraure 17. Early stage. Simulated fuselage-flap configuration. The solution is shown at $t=0.1$, 0.3 and 0.5 . (a) $\delta=0.1,(b) 0.02$.

Throughout this stage it is still possible to think of each half-span structure as consisting of three individual vortices connected by stretched curve segments. The numbers of turns in each vortex increases with time as the roll-up proceeds. The vortices are eventually brought into close proximity and some portions of the curve that were previously distant approach one another closely. The curve at $t=4$ (figure $20 d$ ) has two small intervals of extremely large curvature on the outer turns of the tip and flap vortices.

The results in figures 19 and 20 could have been obtained without using a point insertion technique only by using a much larger fixed value of $N$, at greater computational expense. For example, figure 21 (a) shows the result at $t=4$ using the fixed value $N=1000$. Even with this large value of $N$, a difficulty occurs on the curve's most highly stretched portion, between the tip vortex and the flap vortex. This part of the curve is depleted of vortex blobs as time progresses and eventually, the computed curve intersects itself as shown in figure $21(a)$. To overcome this loss of resolution, the following procedure was adopted. A mesh parameter $\epsilon$ was given as input. The condition $\left|z_{j}-z_{j+1}\right| \leqslant \epsilon$ was enforced at every time-step by inserting a 
(a)

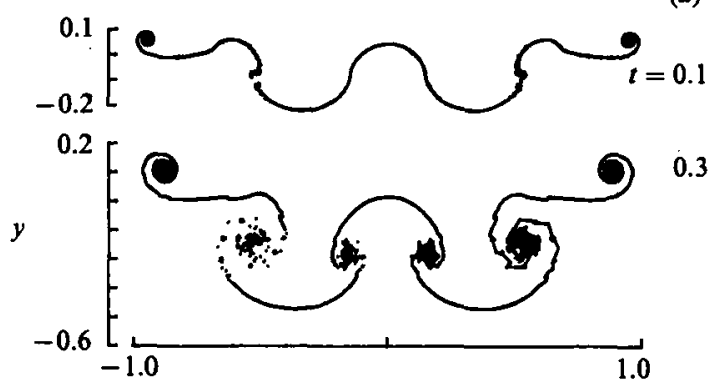

(b)

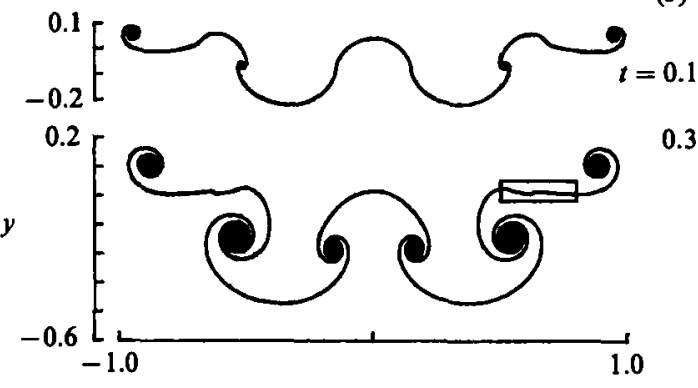

(c)

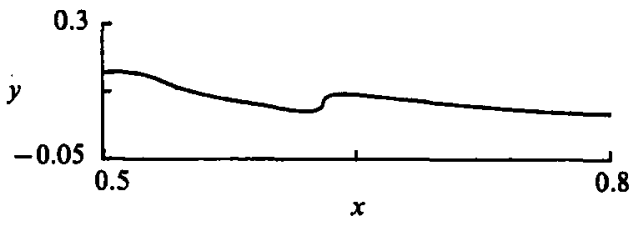

Figure 18. Computed solution for $\delta=0.005$ at $t=0.1$ and 0.3 . (a) 7-digit arithmetic (VAX single precision). (b) 14-digit arithmetic (CRAY single precision). (c) Closeup view of the wave shown in the box of $(b)$, suggesting the formation of a fourth vortex spiral.

new computational point into any interval that had stretched enough to violate the condition. The inserted point was chosen to correspond to the curve parameter value $\alpha=\frac{1}{2}\left(\alpha_{j}+\alpha_{j+1}\right)$ and its coordinates were determined by cubic polynomial interpolation with respect to $\alpha$, using the four neighbouring vortex blobs. The interpolating curve that appears in this section's figures is a plot of this piecewise cubic polynomial. We chose this interpolant here because it requires only that the curve be continuous in the parameter $\alpha$. Higher-order spline interpolation is not called for at this stage of the investigation since it requires a certain degree of smoothness in the underlying curve, which may not actually be attained.

It was checked that convergent results are obtained by decreasing the value of $\epsilon$. This is illustrated in figure $21(b)$ which plots the curve at $t=4$ that was obtained using the value $\epsilon=0.2$ with $N=200$ initially. This should be compared with the solution in figure 19 for which $\epsilon=0.04$. The point insertion technique enabled us to control the $\alpha$-discretization error for a long time, at reasonable computational expense. For example, the computation shown in figure 19 took about 2 hours to run in single-precision arithmetic on a VAX 8600 computer. Computations beyond $t=4$, presented below, used double-precision arithmetic, not because of amplification of round-off error perturbations as occurs for smaller values of $\delta$, but because some 


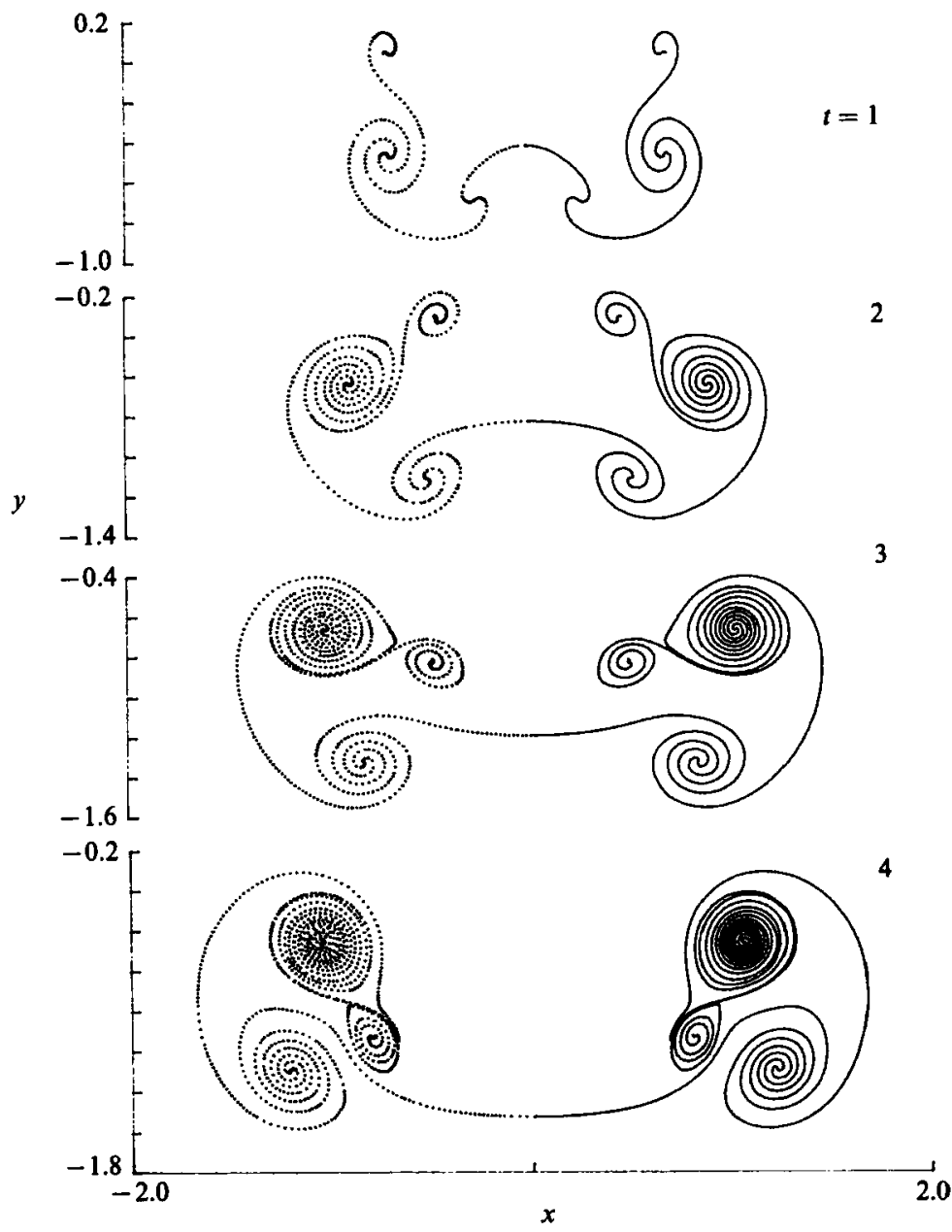

Figure 19. Middle stage. Solution plotted over the time interval $1 \leqslant t \leqslant 4$ using $\delta=0.1$. An adaptive point insertion technique was used to maintain accuracy. The number of vortex blobs in the solution was $N=200$ at $t=0$ and reached $N=971$ at $t=4$.

intervals were bisected so many times that the $\alpha_{j}$ values describing the endpoints were separated by less than the single-precision round-off unit.

In figure 22 we present closeup views of the solution computed over the time interval $1 \leqslant t \leqslant 4$ using $\delta=0.05$, half the previous smoothing parameter value. The relative sizes, shapes and positions of the vortices are quite similar to those in figure 20 , computed using the larger value $\delta=0.1$. The main difference is that at a given time, each vortex has more turns in the $\delta=0.05$ calculation than in the $\delta=0.1$ calculation. Also, the curve computed with the smaller value of $\delta$ develops large curvature sooner (e.g. compare figures $20 \mathrm{c}$ and $22 \mathrm{c}$, at $t=3$ ). In both calculations at $t=4$ (figures $20 d$ and $22 d$ ) the curve portion connecting the flap and fuselage vortices is very strongly stretched. Also, the outer turn of the tip vortex is beginning to be wrapped around the flap vortex. A similar feature occurred in contour-dynamics calculations for the merger of like-signed regions of constant vorticity (Overman \& Zabusky 1982). 

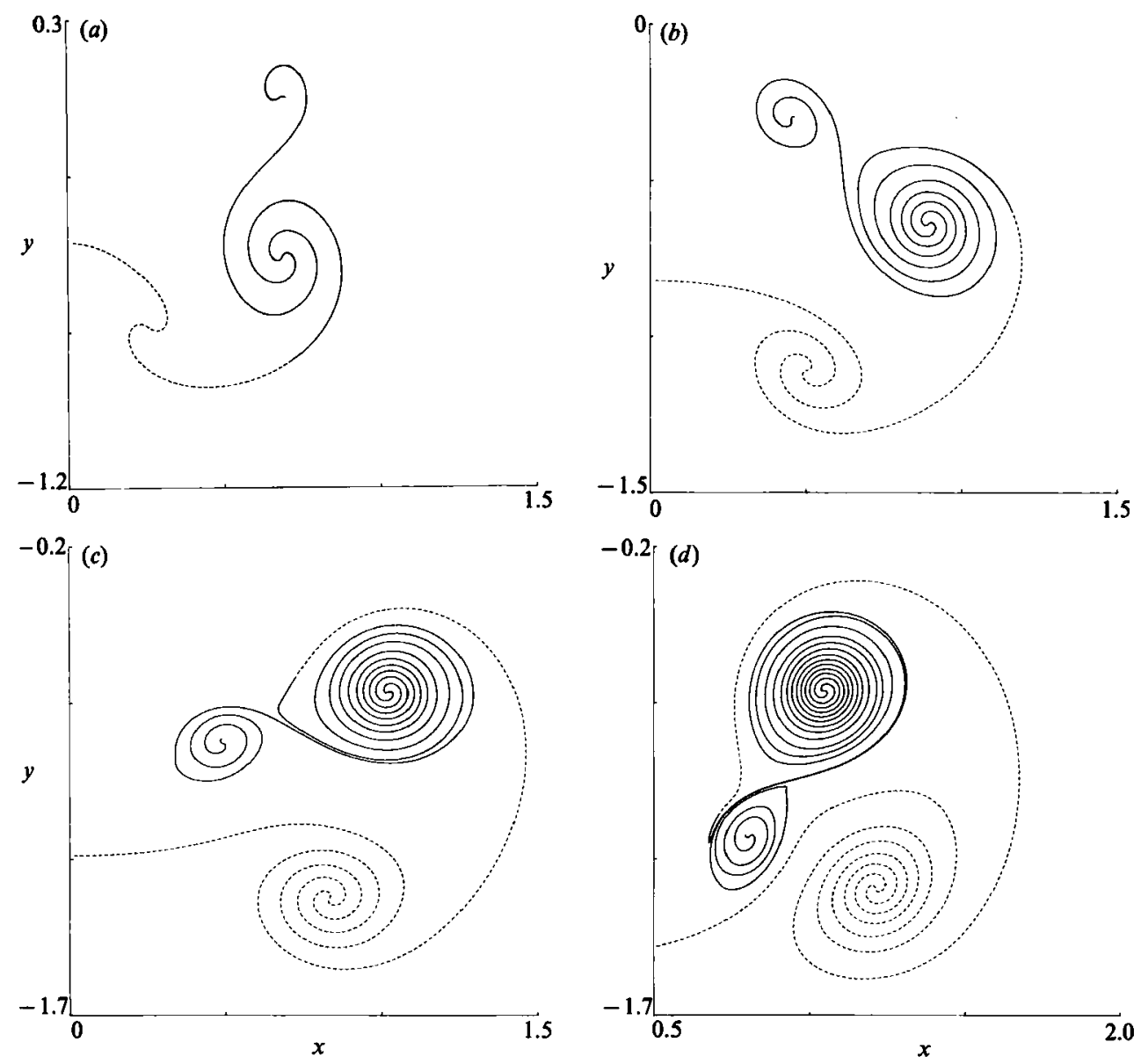

FIGURE 20. Closeup views of the right side for the $\delta=0.1$ calculation. The point insertion parameter was set to $\epsilon=0.04$. (a) $t=1, N=254$. (b) $t=2, N=455$. (c) $t=3, N=711$. (d) $t=4, N=971$. The dashed (solid) portion of the curve corresponds to points which had negative (positive) initial vortex-sheet strength.

\subsection{Late stage}

The capture by a vortex of a previously remote portion of the curve signals the start of more complicated interactions. Figure 23 shows the evolution using the value $\delta=0.1$ over the time interval $5 \leqslant t \leqslant 8$. Closeup views of the right side are given in figure 24. At $t=8$ the solution contains $N=10604$ vortex blobs and the final time step $(\Delta t=0.02)$ of the calculation took about 7 hours to run on a VAX 8600 computer. A $16 \mathrm{~mm}$ film of the evolution was made and viewing it helped to sort out the dynamics. The processes occurring are not presently well understood; here we shall attempt to describe in words some important features of the dynamics depicted in figures 23 and 24.

The tip vortex completes more than one full revolution around the flap vortex between $t=5$ and 8 . The fuselage vortex also rotates from its position below the flap vortex at $t=4$ (figure $20 d$ ) to above the flap vortex at $t=8$ (figure $24 d$ ). This change in relative position causes the flap and fuselage vortices to propagate inward at late times, towards the symmetry line $x=0$ (figure 23). Thus, the structure's lateral extent stops increasing during the late stage, in contrast to the steady increase that 

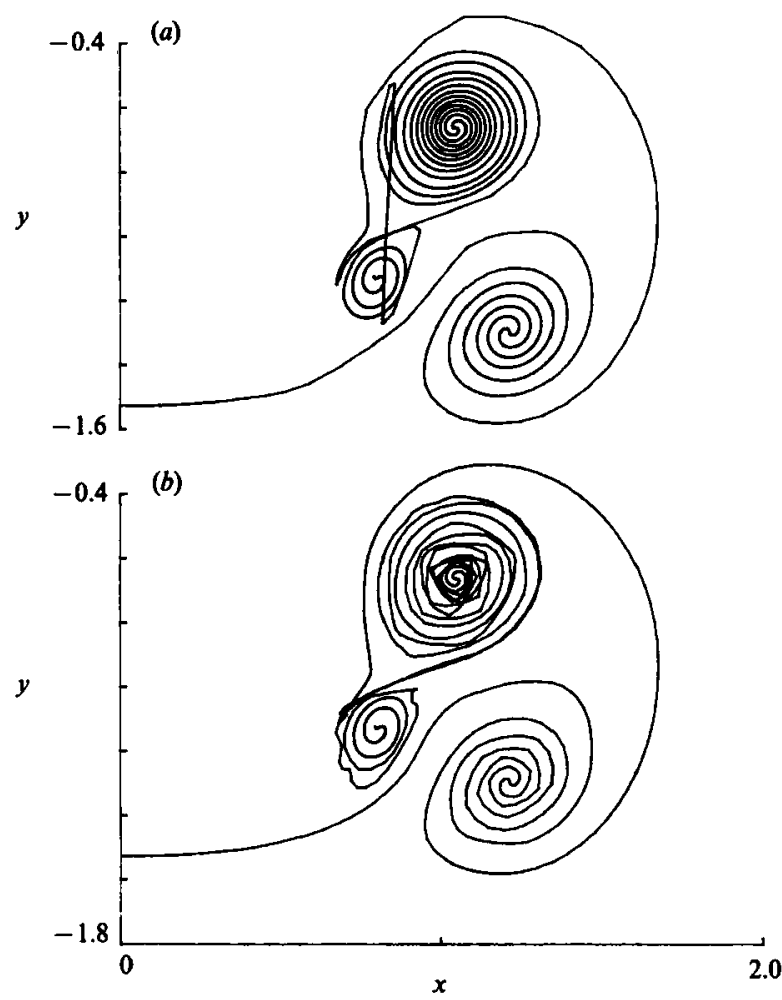

Figure 21. The effect of the $\alpha$-mesh upon the computational accuracy at $t=4$. (a) $N=1000$, fixed throughout the computation. The computation in $(b)$ used the point insertion technique described in the text with the starting value $N=200$. (b) $\epsilon=0.2, N=290$. Compare this with figure 19 at $t=4$ which used $\epsilon=0.04$ and had $N=971$.

occurred for $1 \leqslant t \leqslant 4$ (figure 19). However, if one includes the line segment connecting the two half-span regions, then the structure's vertical extent increases steadily during the entire evolution.

The tip vortex plays an important role in splitting off a portion of the fuselage vortex between times $t=5$ and 6 . The tip vortex is swept around the larger flap vortex and near $t=5$ it collides with, and begins to strongly deform, the fuselage vortex (figure $24 a$ ). The aftermath, shown in figure $24(b)$ at $t=6$, is that the tip vortex separates the fuselage vortex core to its right from another region of dashed lines to its left. This latter region becomes further separated from the fuselage vortex core at $t=7$ (figure 24c). Throughout, the curve remains continuous although various portions are highly stretched.

At $t=8$ (figure $24 d$ ), the tip vortex core is greatly reduced from its size at $t=5$. The flap and fuselage vortex cores are also smaller, although each can still be identified as substantial regions of roughly concentric turns. The reduction in core size occurs because each vortex has been stripped of its outer turns. A large portion of the curve in figure $24(d)$ lies away from the cores, in long sweeping roughly one-dimensional fronts. These fronts consist of many distinct almost parallel curve portions that are very closely spaced.

Figure 25 shows a closeup view of the $\delta=0.05$ calculation at $t=5$. Here, the collision of the tip vortex with the fuselage vortex is more advanced than with $\delta=0.1$ at $t=5$ (compare with figure 24a). With the smaller value of $\delta$, the rotation rate 

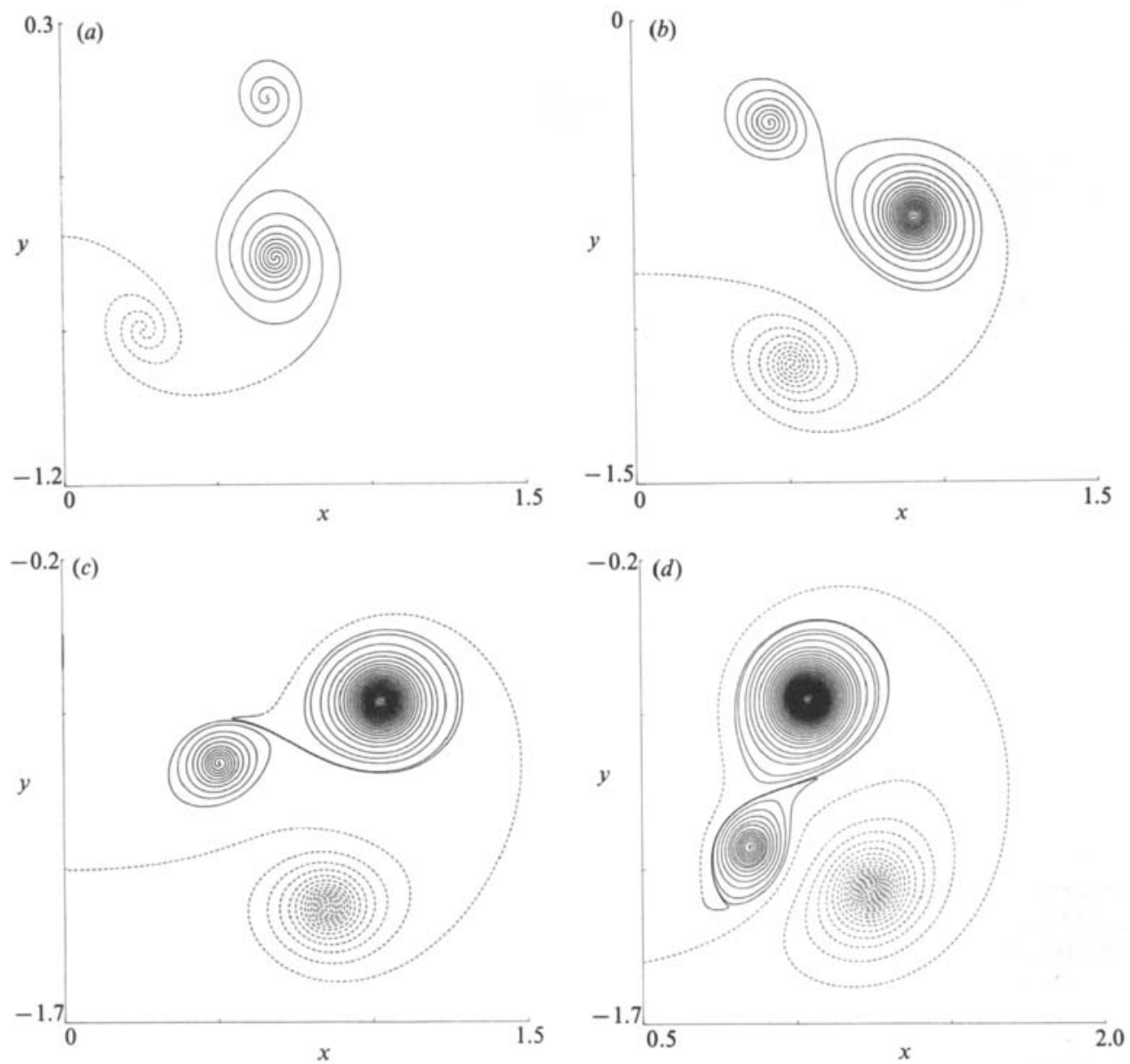

Fiadre 22. Closeup views of the right side for the $\delta=0.05$ calculation. The point insertion parameter was set to $\epsilon=0.013$. (a) $t=1, N=1078$. (b) $t=2, N=2403$. (c) $t=3, N=3680$. (d) $t=4, N=5659$. The dashed (solid) portion of the curve corresponds to points which had negative (positive) initial vortex-sheet strength.

around the strong flap vortex is larger, causing the tip vortex to travel faster. The tip vortex also accelerates near $t=5$, owing to the local jet-like flow created by its two oppositely rotating neighbours. This $\delta=0.05$ computation used the time-step $\Delta t=0.0125$ and the point insertion parameter value $\epsilon=0.013$. The solution started with $N=200$ vortex blobs, increasing to $N=8838$ at $t=5$. The computation used about two hours of c.p.u. time on the CRAY2 computer. Computations over longer time intervals with smaller values of $\delta$ are certainly desirable and feasible, although significantly more c.p.u. time would be required.

It is emphasized again that the plotted results have negligible discretization error and that what is being seen here are the true dynamics of the $\delta$-equation for these particular values of $\delta$. As already mentioned, the late-time results should be viewed as possible hints about the actual vortex sheet's evolution. If here too, as documented at $t=4$ for the elliptically loaded wing problem, the computed curve converges as $\delta \rightarrow 0$, then these results at least describe the overall vortex-sheet shape and the relative vortex positions. Even with the relatively large values of $\delta$ used in the late 

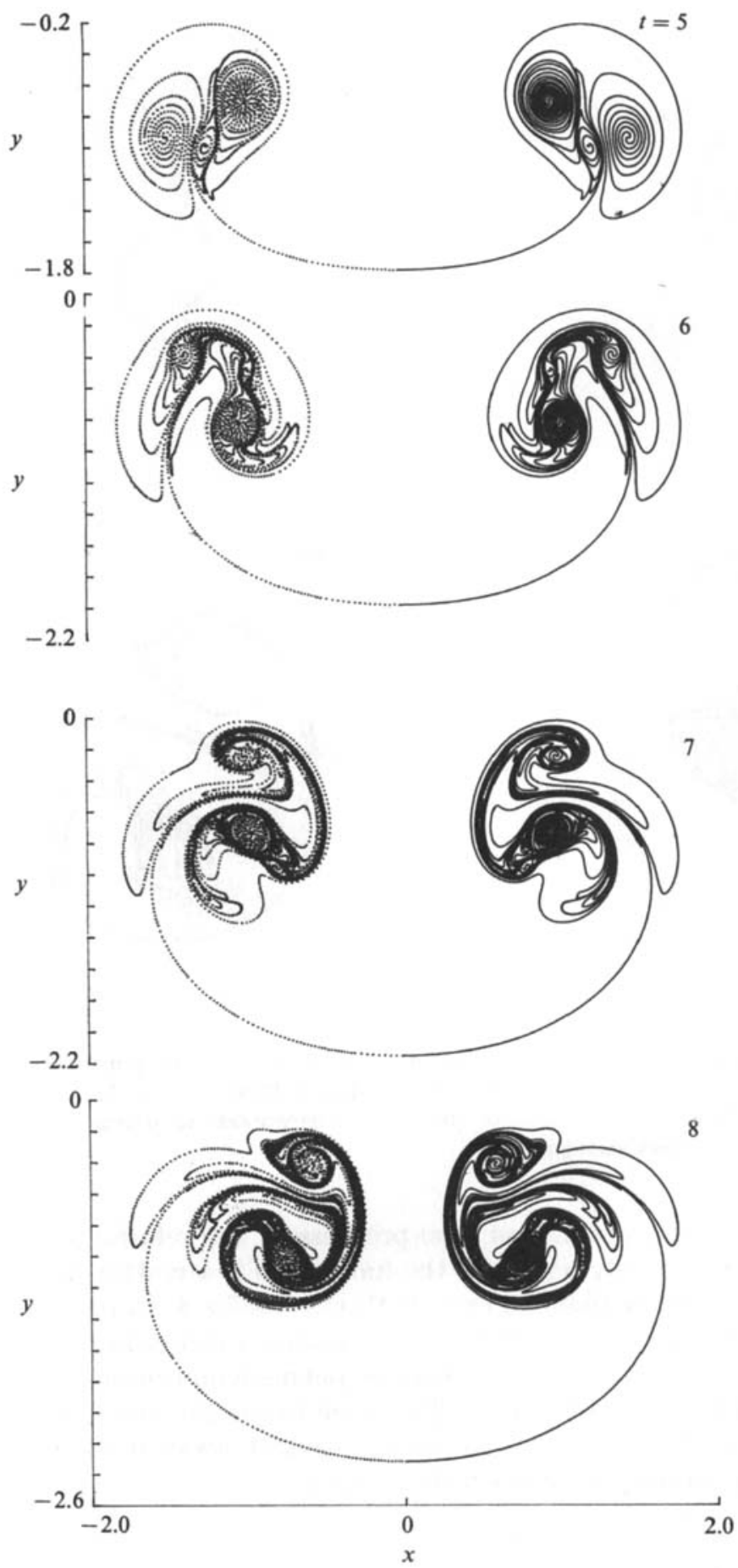

Froure 23. Late stage. Evolution over the time interval $5 \leqslant t \leqslant 8$ for the $\delta=0.1$ computation. 

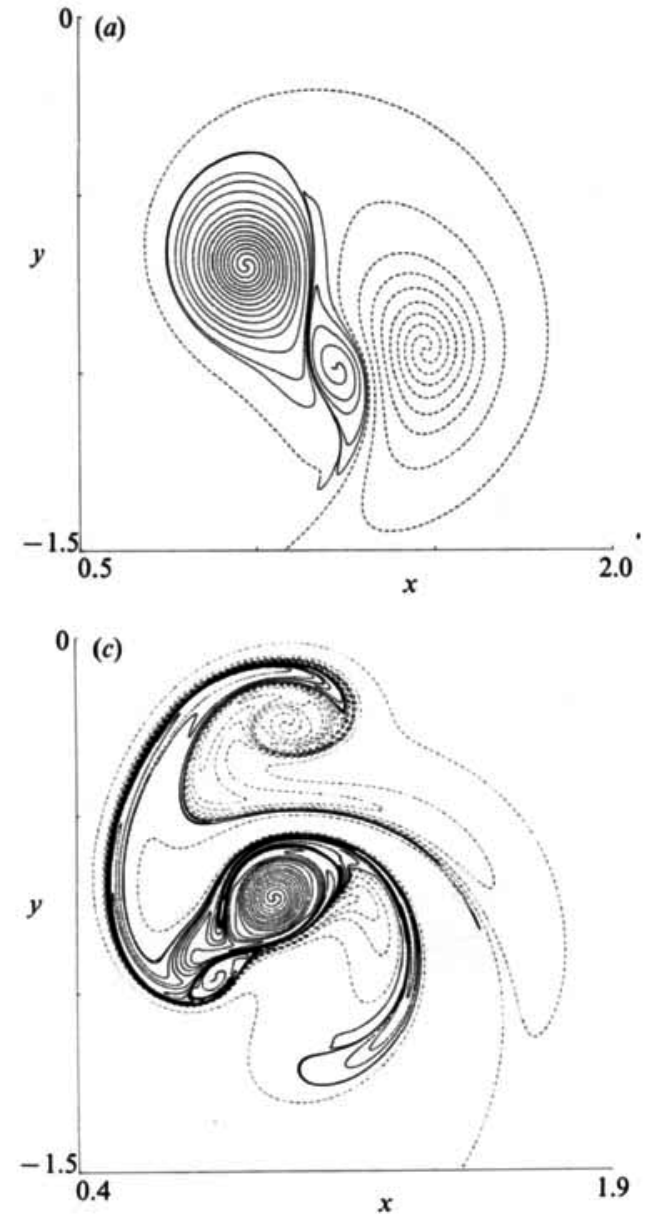

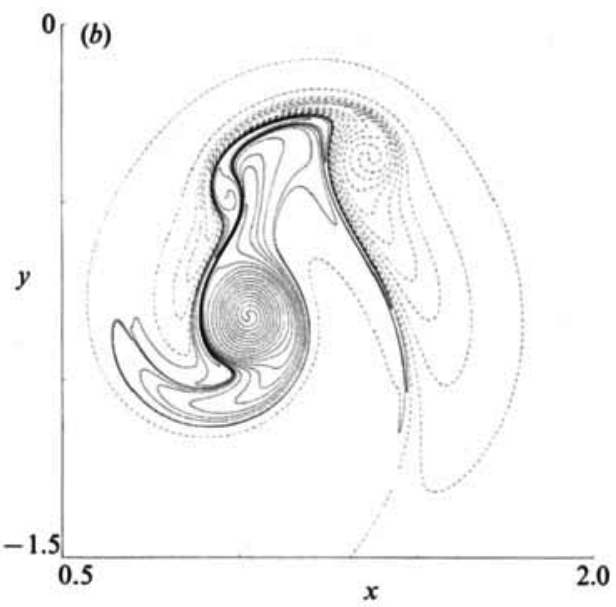

$0_{(d)}$

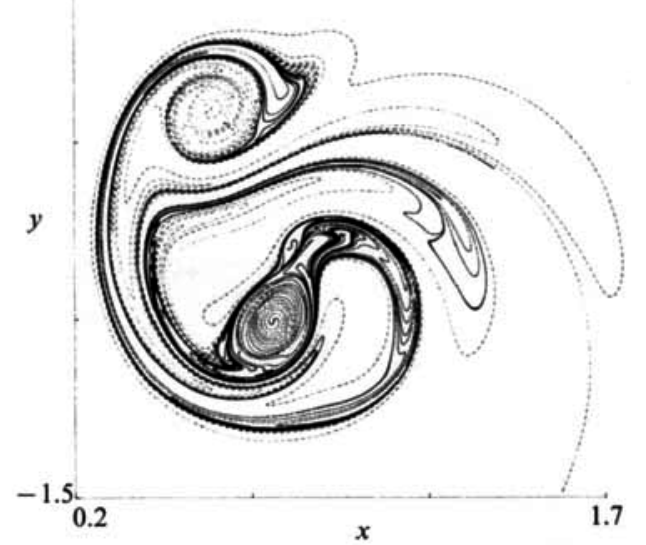

Figure 24. Closeup views of the solution with $\delta=0.1$ (figure 23). The point insertion parameter was set to $\epsilon=0.04$. (a) $t=5, N=1422$. (b) $t=6, N=2580$. (c) $t=7, N=5395$. (d) $t=8$, $N=10604$. The dashed (solid) portion of the curve corresponds to points which had negative (positive) initial vortex sheet strength.

stage, quite small scales develop as time progresses. The relation between this fine structure and what actually occurs in the limit $\delta \rightarrow 0$ is a matter for speculation at present. We note however that DiPerna \& Majda $(1987 a, b)$ have recently begun to study such questions in the context of measure-valued solutions of the Euler equations. They discuss a new concentration phenomenon occurring for the Euler equations and suggest that it may be illustrated in the present computation where separate portions of the curve become tightly packed, away from the vortex cores. This interesting possibility deserves further study.

\section{Closing remarks}

A method for computing vortex-sheet evolution in the Trefftz plane has been presented. Results were obtained for the elliptically loaded wing problem and for a simulated fuselage-flap configuration. The key idea is to desingularize the Cauchy principal value integral which defines the velocity of a Lagrangian point on the vortex 


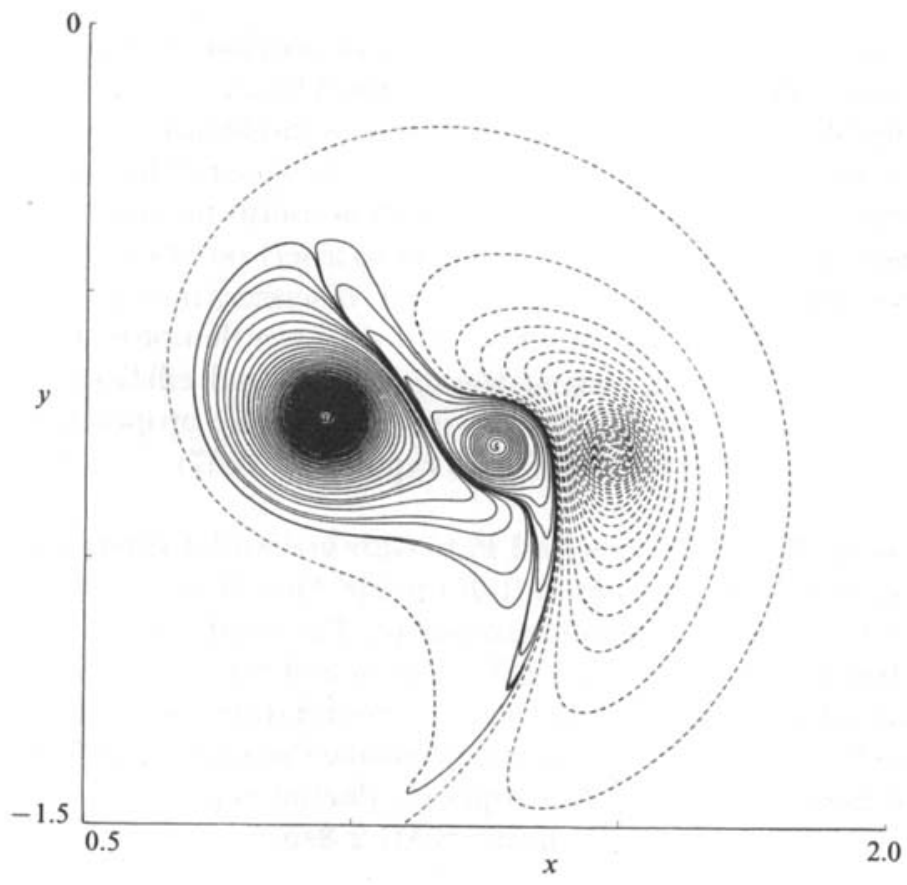

Figure 25. Closeup view of the solution with $\delta=0.05$ at $t=5(N=8838)$. The point insertion parameter was set to $\epsilon=0.013$.

sheet. The approach was heuristically motivated by considering that another Cauchy principal value integral, the Hilbert transform, can be alternatively defined via the boundary values of a harmonic function in the upper half-plane. The desingularized vortex-sheet equation is numerically tractable and the discretization was demonstrated to converge under mesh refinement for a fixed value of the smoothing parameter $\delta>0$. The smoothing parameter controls the amount of detail that is present in the computed solution. Numerical evidence was also presented indicating that the desingularized solutions converge to a well-defined spiral as $\delta \rightarrow 0$. Away from the spiral's centre, the convergence is uniform and the error behaves like an asymptotic power series in the smoothing parameter $\delta$. These conclusions about convergence are based on experimental computational evidence and it is certainly desirable that they be placed on a more rigorous footing in the future.

The desingularization used here was chosen for heuristic mathematical reasons and numerical convenience. Its physical significance is unclear at present. Physically motivated desingularizations such as the Navier-Stokes equations with Reynolds number $R e$, and the Euler equations for a vorticity field characterized by a thickness $d$, may also be considered (e.g. Weston \& Liu 1982). It is not known whether the three limits $\delta \rightarrow 0, R e^{-1} \rightarrow 0$ and $d \rightarrow 0$ coincide for the type of problem considered here. It would be interesting to know how closely these sequences approach one another (or how quickly they diverge) for non-zero smoothing-parameter values. Clearly, the practical significance of our results would be enhanced if it could be shown that the solution of the $\delta$-equation with $\delta>0$ approximates in some sense a solution of either the Navier-Stokes equations with some $R e^{-1}>0$ or the Euler equations with $d>0$.

A significant consideration for the present method is its expense - with $N$ vortex blobs, the computational work per time-step is $O\left(N^{2}\right)$. Even so, highly resolved 
solutions over long time intervals were obtained and new details of the vortex sheet's evolution were suggested which had not appeared in previous numerical studies. Care was taken to ensure that the time and spatial discretization error in the computed results was negligible. In accomplishing this, it was important to place the computational elements where they were needed. Uniformly spacing the vortex blobs in the parameter $\alpha$ crowds them towards the tip, which is useful for the elliptically loaded wing. For the fuselage-flap problem it was better to insert points adaptively in regions where the curve was stretching. The spatial discretization and time-stepping strategies used here are simple to program and we have demonstrated their ability to yield accurate solutions. Increasing the computational efficiency is a topic for future research and several possibilities have been recently proposed (e.g. Anderson 1986; Carrier, Greengard \& Rokhlin 1986; Tryggvason 1987).

I thank Professors Marsha Berger and Paul Garabedian for interesting discussions during the course of this work. I am grateful to Dr Alex Woo and to the referees for suggestions on improving the original manuscript. The computations were performed at the Courant Institute of Mathematical Sciences and the University of Minnesota. I gratefully acknowledge the National Science Foundation for a grant of supercomputer time through the Office of Advanced Scientific Computing (DMS 8414101) and for Postdoctoral Research Fellowship support. Partial support also came from the NASA-Ames Research Center under grant NAG 2-345.

\section{REFERENCES}

Anderson, C. R. 1985 A vortex method for flows with slight density variations. J. Comp. Phys. $61,417$.

Anderson, C. R. 1986 A method of local corrections for computing the velocity field due to a distribution of vortex blobs. J. Comp. Phys. 62, 111.

BAKER, G. R. 1979 The "Cloud in Cell" technique applied to the roll up of vortex sheets. J. Comp. Phys. 31, 76.

Batchelor, G. K. 1967 An Introduction to Fluid Dynamics. Cambridge University Press.

BIRkноF, G. 1962 Helmholtz and Taylor instability. Proc. Symp. Appl. Math. XIII A.M.S.P. 55.

Carrier, J., Greengard, L. \& Rokrlin, V. 1986 A fast adaptive multipole algorithm for particle simulations. Yale Univ. Comp. Sci. Res. Rep. YALEU/DCS/RR-496.

Chorin, A. J. \& Bernard, P. S. 1973 Discretization of a vortex sheet, with an example of roll-up. J. Comp. Phys. 13, 423.

DiPerna, R. J. \& Majda, A. $1987 a$ Oscillations and concentrations in weak solutions of the incompressible fluid equations. Commun. Math. Phys. 108, 667.

DiPerna, R. J. \& Majda, A. $1987 b$ Concentrations in regularizations for 2-D incompressible flow. Commun. Pure Appl. Math. 40, 301.

Donaldson, C. DuP., SNedeker, R. S. \& Sullivan, R. D. 1974 Calculation of aircraft wake velocity profiles and comparison with experimental measurements. J. Aircraft 11, 547.

Fink, P. T. \& SoH, W. K. 1978 A new approach to roll-up calculations of vortex sheets. Proc. $R$. Soc. Lond. A 362, 195.

Guiradd, J. P. \& ZeYtounian, R. Kh. 1977 A double-scale investigation of the asymptotic structure of rolled-up vortex sheets. J. Fluid Mech. 79, 93.

Hoeijmakers, H. W. M. 1983 Computational vortex flow aerodynamics. AGARD Conf. Proc. no. 342 , paper 18 , also $N L R M P 83017 \mathrm{U}$.

HoEIJMAK ERS, H. W. M. 1986 Numerical simulation of vortical flow. In lecture series Introduction to Vortex Dynamics, von Karman Inst. Fluid Dyn., Belgium, also NLR MP 86032 U. 
HoeiJmakers, H. W. M. \& VaAtstra, W. 1983 A higher-order panel method applied to vortex sheet roll-up. AIAA J. 21, 516.

KADEN, H. 1931 Aufwicklung einer unstabilen Unstetgkeitsfläche. Ing. Arch. 2, 140. (English transl., R.A.E. Library Trans. 403.)

KRASNY, R. 1986a A study of singularity formation in a vortex sheet by the point vortex approximation. J. Fluid Mech. 167, 65.

Krasny, R. $1986 b$ Desingularization of periodic vortex sheet roll-up. J. Comp. Phys. 65, 292.

Kuwahara, K. \& Takami, H. 1973 Numerical studies of two-dimensional vortex motion by a system of point vortices. J. Phys. Soc. Japan 34, 247.

Moore, D. W. 1971 The discrete vortex approximation of a finite vortex sheet. Calif. Inst. Tech. Rep. AFOSR-1804-69.

Moore, D. W. 1974 A numerical study of the roll-up of a finite vortex sheet. J. Fluid Mech.63, 225.

Mooke, D. W. 1975 The rolling up of a semi-infinite vortex sheet. Proc. R. Soc. Lond. A 345, 417.

Moore, D. W. 1976 The stability of an evolving two-dimensional vortex sheet. Mathematika 23, 35.

Moore, D. W. \& Saffman, P. G. 1973 Axial flow in laminar trailing vortices. Proc. R. Soc. Lond. A 333, 491.

Murman, E. M. \& Stremel, P. M. 1982 A vortex wake capturing method for potential flow calculations. AIAA paper $82-0947$.

Overman II, E. A. \& ZabUSKy, N. I. 1982 Evolution and merger of isolated vortex structures. Phys. Fluids 25, 1297.

PulLIN, D. I. 1978 The large-scale structure of unsteady self-similar rolled-up vortex sheets. $J$. Fluid Mech. 88, 401.

Pulurs, D. I. \& Phrlurs, W. R. C. 1981 On a generalization of Kaden's problem. J. Fluid Mech. 104, 45.

Rosenhead, L. 1931 The formation of vortices from a surface of discontinuity. Proc. R. Soc. Lond. A $134,170$.

Rotr, N. 1956 Diffraction of a weak shock with vortex generation. J. Fluid Mech. 1, 111.

SaffMan, P. G. \& Baker, G. R. 1979 Vortex interactions. Ann. Rev. Fluid Mech. 11, 95.

Sмiтн, J. H. B. 1986 Vortex flows in aerodynamics. Ann. Rev. Fluid Mech. 18, 221.

Stmin, E. M. \& WeIss, G. 1971 Introduction to Fourier Analysis on Euclidean Spaces. Princeton University Press.

TAKami, H. 1964 A numerical experiment with discrete vortex approximation with reference to the rolling up of a vortex sheet. Dept. Aero \& Astron., Stanford Univ. SUDAER 202.

Trygavason, G. 1987 Simulation of vortex sheet roll-up by vortex methods. J. Comp. Phys. (to appear).

Whston, R. P. \& LuU, C. H. 1982 Approximate boundary condition procedure for the twodimensional numerical solution of vortex wakes. AIAA paper 82-0951.

WESTWATER, F. L. 1935 Rolling up of the surface of discontinuity behind an aerofoil of finite span. Aero. Res. Counc. $R \& M .1692$. 